\title{
The minimal linear $\sigma$ model for the Goldstone Higgs
}

\author{
F. Feruglio, ${ }^{a}$ M.B. Gavela, ${ }^{b}$ K. Kanshin, ${ }^{a}$ P.A.N. Machado, ${ }^{b}$ S. Rigolin $^{a}$ and S. Saa ${ }^{b}$ \\ ${ }^{a}$ Dipartimento di Fisica e Astronomia 'G. Galilei', Università di Padova, \\ INFN, Sezione di Padova, \\ Via Marzolo 8, I-35131 Padua, Italy \\ ${ }^{b}$ Departamento de Fúsica Teórica and Instituto de Física Teórica, IFT-UAM/CSIC, \\ Universidad Autónoma de Madrid, \\ Cantoblanco, 28049, Madrid, Spain \\ E-mail: feruglio@pd.infn.it, belen.gavela@uam.es, kanshin@pd.infn.it, \\ pedro.machado@uam.es, stefano.rigolin@pd.infn.it, sara.saa@uam.es
}

AbStRaCT: In the context of the minimal $\mathrm{SO}(5)$ linear $\sigma$-model, a complete renormalizable Lagrangian -including gauge bosons and fermions- is considered, with the symmetry softly broken to $\mathrm{SO}(4)$. The scalar sector describes both the electroweak Higgs doublet and the singlet $\sigma$. Varying the $\sigma$ mass would allow to sweep from the regime of perturbative ultraviolet completion to the non-linear one assumed in models in which the Higgs particle is a low-energy remnant of some strong dynamics. We analyze the phenomenological implications and constraints from precision observables and LHC data. Furthermore, we derive the $d \leq 6$ effective Lagrangian in the limit of heavy exotic fermions.

KeYwords: Higgs Physics, Sigma Models

ARXiv EPrint: 1603.05668 


\section{Contents}

1 Introduction 1

2 The $\mathrm{SO}(5) / \mathrm{SO}(4)$ scalar sector $\quad 4$

2.1 The scalar potential 5

$\begin{array}{lll}2.2 & \text { Scalar-gauge boson couplings } & 7\end{array}$

$\begin{array}{lll}2.3 & \text { Renormalization and scalar tree-level decays } & 8\end{array}$

3 Fermionic sector $\quad 11$

4 Phenomenology $\quad \mathbf{1 5}$

$\begin{array}{lll}4.1 & \text { Bounds from Higgs measurements } & 15\end{array}$

$\begin{array}{lll}4.2 & \text { Precision electroweak constraints } & 16\end{array}$

$\begin{array}{lll}4.2 .1 & S, T \text { and } g_{L}^{b} & 16\end{array}$

$\begin{array}{lll}4.3 & \text { Higgs and } \sigma \text { coupling to gluons } & 25\end{array}$

4.4 Higgs and $\sigma$ decay into $\gamma \gamma \quad 28$

$5 \quad$ The $\sigma$ resonance at the $\mathrm{LHC} \quad 31$

$6 \quad d \leq 6$ fermionic effective Lagrangian $\quad 34$

$\begin{array}{lll}7 & \text { Conclusions } & 35\end{array}$

$\begin{array}{ll}\text { A Coleman-Weinberg potential } & 40\end{array}$

\section{Introduction}

The discovery of the Higgs boson has confirmed the simplest possible picture of electroweak symmetry breaking and mass generation. As predicted long ago by the Standard Model of particle physics (SM), the Higgs mechanism splits an elementary doublet of scalar particles into an unphysical sector, providing the longitudinal polarization to the vector bosons $W$ and $Z$, and a single physical spin zero particle. For a long time this picture has been questioned, specially in connection to the naturalness problem, also called "electroweak hierarchy problem", that is the stability of the electroweak scale against quantum corrections and its smallness compared to other higher scales to which the Higgs field may be sensitive, if such new scales of physics do exist in nature. The tension would be manifest in the Higgs mass being lower than those putative scales.

The challenge raised by the lightness of the Higgs mass becomes increasingly pressing as long as no firm signal of beyond the Standard Model (BSM) physics appears in new data. The resistance to accept extreme fine-tunings has been historically most fruitful, 
prompting the identification of new symmetries to justify dynamically scales smaller than the characteristic overall scale of a given theory; a magnificent example is for instance the prediction of the charm particle [1] and its mass [2]. The observed light Higgs mass poses a similar conundrum.

Moreover the Higgs boson of the SM would represent the unique example of elementary spin zero particle in nature, while in other known phenomena of spontaneous symmetry breaking its role is played by composite excitations. Supersymmetry would justify elementary scalars, actually one copy for each known fermion, but no direct or indirect hints of them has been found so far. In fact, the only degrees of freedom found in nature prior to the Higgs discovery which may originate from scalar fields were Goldstone bosons: the longitudinal components of the electroweak gauge bosons. This suggested decades [3-7] ago a dynamical nature for the Higgs particle as a pseudo-Nambu-Goldstone boson (PNGB) which would justify a light Higgs. All components of the Higgs multiplet would then share a common Goldstone origin, providing a beautifully homogeneous picture. In the initial proposal the Higgs originated from one of the Goldstone bosons produced in the spontaneous breaking of a high-energy SU(5) invariant strong dynamics. Recent attempts tend to start instead from a $\mathrm{SO}(5)$ symmetry $[8,9]$ spontaneously broken to $\mathrm{SO}(4)$ at some high scale $\Lambda$, producing at this stage an ancestor of the Higgs particle in the form of one of the resulting Goldstone bosons, with characteristic scale $f$ and $\Lambda \leq 4 \pi f$ [10]. The coset $\mathrm{SO}(5) / \mathrm{SO}(4)$ represents the minimal possibility to interpret the Higgs as a pseudo-Goldstone boson in the presence of a custodial symmetry.

The fermionic couplings of the $\mathrm{SO}(5)$ invariant sector to the SM fermions and gauge bosons give an additional - generally soft — breaking of $\mathrm{SO}(5)$ resulting in a potential for the Goldstone Higgs. Its minimum breaks spontaneously the electroweak symmetry at a scale $v$, which phenomenologically needs to differ from $f$, and gives a mass to the Higgs particle. Moreover, this type of theories [11] proposes naturally a seesaw-like mechanism for quarks and leptons, whose masses would be inversely proportional to the heavy fermion mass scale. It is most suggestive that the seesaw mechanism would not then be reduced to the realm of neutrino masses - for which it is the best candidate theory - but it would be instead the universal pattern behind all fermion masses.

A Goldstone-boson parenthood for the Higgs is not exclusive of those models, often called "composite Higgs" models, but is also embedded in other constructions such as "little Higgs" models, extra-dimensional scenarios and others. In concrete models the spectra of exotic fermions are directly related to the light fermion masses - in particular the top mass - and the Higgs mass. The values of these masses generally require a spectrum with some exotic fermion masses below the $\mathrm{TeV}$ scale, a fact often in tension with experimental searches $[12,13]$. It is interesting to clarify the degree of fine-tuning that the models require, in view of the electroweak hierarchy problem.

Most of the literature on composite Higgs models based on $\mathrm{SO}(5)$ assumes from the start a strong dynamics and uses an effective non-linear formulation of the model(s) $[9,14-20]$. This approach has the advantage of being completely general, offering a parametrization of all possible ultraviolet completions for the symmetry group chosen. At the same time one of its limitations is that it is applicable only in a finite 
domain of energies. Here instead we construct a complete renormalizable model which in its scalar part is a linear sigma model including a new scalar particle $\sigma$, singlet under the gauge group. This will allow to gain intuition on the dependence on the ultraviolet (UV) completion scale of the model, by varying the $\sigma$ mass: a light $\sigma$ particle corresponds to a weakly coupled regime, while in the high mass limit the theory should fall back onto an usual effective non-linear construction. Our complete renormalizable model can thus be considered either as an ultimate model made out of elementary fields, or as a renormalizable version of a deeper dynamics, much as the linear $\sigma$ model [21] is to QCD. One former attempt in this direction [22] did not fully take into account and computed the impact of the fermionic sector on the main phenomenological observables, see also refs. [23, 24].

While the choice of the minimal bosonic sector is clear, there is a number of possible choices for the fermionic sector. The option explored in this paper assumes heavy fermions in vectorial representations of $\mathrm{SO}(5)$, in contrast to models where the SM left doublets are embedded in $\mathrm{SO}(5)$ multiplets [22]. Direct couplings between SM fermions and the heavy fermions will be the source of the soft $\mathrm{SO}(5)$ breaking, while the Higgs particle has tree-level couplings only with the exotic fermionic sector, via SO(5)-invariant Yukawa couplings. It will be discussed how the induced Coleman-Weinberg potential requires soft breaking terms to be included in the scalar potential.

The usual SM Higgs sector is now substituted by a Higgs- $\sigma$ sector, correcting the strength of the SM Higgs-gauge boson couplings and opening new interaction channels. The phenomenology of the $\sigma$ production and decay will be also studied, including fermionic and bosonic tree-level and one loop decays (e.g. gluon-gluon and photon-photon). Analysis of present Higgs data will be used to set a constraint on the fine-tuning ratio $v / f$. The contribution of the Higgs, $\sigma$ and the exotic fermions to the oblique $S$ and $T$ parameters will be computed. One aim of the phenomenological study is to clarify the impact of the size of the ultraviolet scale - here represented by the $\sigma$ mass - on the tensions of this type of theories. Particular emphasis will be dedicated to the impact of the $\sigma$ particle on present and future LHC data, produced either via gluon fusion or vector-boson fusion and decaying into a plethora of channels including diphoton final state.

Furthermore, as this paper focuses on the impact of a dynamical $\sigma$ particle, we identify below some of the leading low-energy bosonic operators stemming from the new physics when the exotic heavy fermion sector is integrated out: we determine the dominant effective operators made out of the $\sigma$ field and/or SM fields, as a first step towards the identification of a "benchmark" electroweak effective Lagrangian including a light dynamical Higgs. A non-linear effective Lagrangian should result in the limit of very heavy $\sigma$. An interesting characteristic of the non-linear scenario is that the low-energy physical Higgs field turns out not to be an exact electroweak doublet, and may appear in the effective Lagrangian as a generic SM scalar singlet with arbitrary couplings. The most general effective Lagrangian of this type $[25,26]$ turns out to depend on a plethora of couplings, though. It would be useful to identify the reduced pattern of dominant couplings characteristic of models in which the Higgs is a pseudo-Goldstone boson in that regime [27].

The structure of the paper can be easily inferred from the table of contents. 


\section{The $\mathrm{SO}(5) / \mathrm{SO}(4)$ scalar sector}

The complete Lagrangian can be written as the sum of three terms describing respectively the pure gauge, scalar and fermionic sectors,

$$
\mathcal{L}=\mathcal{L}_{\text {gauge }}+\mathcal{L}_{\text {scalar }}+\mathcal{L}_{\text {fermion }}
$$

where $\mathcal{L}_{\text {gauge }}$ reduces to the SM gauge kinetic terms. This section discusses in detail the scalar sector and its interactions, while the study of the fermionic sector is deferred to the next section.

In order to define the linear $\sigma$ model corresponding to an $\mathrm{SO}(5)$ symmetry spontaneously broken to $\mathrm{SO}(4)$, let us consider a real scalar field $\phi$ in the fundamental representation of $\mathrm{SO}(5)$. Three among its five components will be ultimately associated with the longitudinal components of the SM gauge bosons - denoted below by $\pi_{i}, i=1,2,3$, while the other two will correspond to the Higgs particle $h$ and to an additional scalar $\sigma$, respectively. For simplicity the results will be often presented in the unitary gauge (u.g.), in which $\pi_{i}=0$ :

$$
\phi=\left(\pi_{1}, \pi_{2}, \pi_{3}, h, \sigma\right)^{T} \stackrel{\text { u.g. }}{\rightarrow} \quad(0,0,0, h, \sigma)^{T} .
$$

The scalar Lagrangian describing the scalar-gauge and the scalar-scalar interactions reads

$$
\mathcal{L}_{\mathrm{s}}=\frac{1}{2}\left(D_{\mu} \phi\right)^{T}\left(D^{\mu} \phi\right)-V(\phi),
$$

where the $\mathrm{SU}(2)_{L} \times \mathrm{U}(1)_{Y}$ covariant derivative is given by

$$
D_{\mu} \phi=\left(\partial_{\mu}+i g \Sigma_{L}^{i} W_{\mu}^{i}+i g^{\prime} \Sigma_{R}^{3} B_{\mu}\right) \phi
$$

and $\Sigma_{L}^{i}$ and $\Sigma_{R}^{i}$ denote respectively the generators of the $\mathrm{SU}(2)_{L}$ and $\mathrm{SU}(2)_{R}$ subgroups of the custodial $\mathrm{SO}(4)$ group contained in $\mathrm{SO}(5)$. The embedding of the gauge group $\mathrm{SU}(2)_{L} \times \mathrm{U}(1)_{Y}$ inside $\mathrm{SO}(5)$, implicitly assumed in eqs. (2.2)-(2.4), is purely conventional. As we will see in section 2.1, both $h$ and $\sigma$ acquire a vacuum expectation value (vev), leaving unbroken an $\mathrm{SO}(4)^{\prime}$ subgroup which is rotated with respect to the group $\mathrm{SO}(4) \approx$ $\mathrm{SU}(2)_{L} \times \mathrm{SU}(2)_{R}$ containing $\mathrm{SU}(2)_{L} \times \mathrm{U}(1)_{Y}$.

For later convenience it is pertinent to introduce the complex notation for the scalar field $\phi$. Denoting by $H(\tilde{H})$ the SM Higgs doublet transforming as $(2,1 / 2)((2,-1 / 2))$ under the $\mathrm{SU}(2)_{L} \times \mathrm{U}(1)_{Y}$ gauge group, a complex scalar field in the fundamental representation of $\mathrm{SO}(5)$ can be defined as

$$
\hat{\phi}=\left(H^{T}, \tilde{H}^{T}, \sigma\right)^{T},
$$

with the convention that the first two entries of this $\mathrm{SO}(5)$ multiplet are $\mathrm{SU}(2)_{L}$ doublets with $+1 / 2$ and $-1 / 2$ eigenvalue of the diagonal $\mathrm{SU}(2)_{R}$ generator, namely

$$
H=\left(\begin{array}{l}
H^{u} \\
H^{d}
\end{array}\right) \quad \stackrel{\text { u.g. }}{\rightarrow} \quad \frac{1}{\sqrt{2}}\left(\begin{array}{l}
0 \\
h
\end{array}\right) \quad, \quad \tilde{H} \equiv i \sigma_{2} H^{*}=\left(\begin{array}{l}
\tilde{H}^{u} \\
\tilde{H}^{d}
\end{array}\right) \quad \stackrel{\text { u.g. }}{\rightarrow} \quad \frac{1}{\sqrt{2}}\left(\begin{array}{l}
h \\
0
\end{array}\right),
$$

while the last component, $\sigma$, is an $\mathrm{SU}(2)_{L}$ and $\mathrm{SU}(2)_{R}$ singlet. The relation between the real and the complex notation is given by

$$
\phi=\frac{1}{\sqrt{2}}\left(-i\left(H^{u}+\tilde{H}^{d}\right), H^{u}-\tilde{H}^{d}, i\left(H^{d}-\tilde{H}^{u}\right), H^{d}+\tilde{H}^{u}, \sqrt{2} \sigma\right)^{T} .
$$




\subsection{The scalar potential}

The most general $\mathrm{SO}(4)$ preserving while $\mathrm{SO}(5)$ breaking renormalizable potential depends a priori on ten parameters. Two of them can be reabsorbed via a redefinition of parameters, ${ }^{1}$ resulting on a Lagrangian dependent on one $\mathrm{SO}(5)$ preserving coupling, $\lambda$, one scale $f$ heralding spontaneous $\mathrm{SO}(5) / \mathrm{SO}(4)$ breaking, and six $\mathrm{SO}(5)$ soft-breaking terms (denoted below $\left.\alpha, \beta, a_{1,2,3,4}\right)$. The Lagrangian in the unitary gauge reads:

$$
V(h, \sigma)=\lambda\left(\sigma^{2}+h^{2}-f^{2}\right)^{2}+\alpha f^{3} \sigma-f^{2} \beta h^{2}+a_{1} f \sigma h^{2}+a_{2} \sigma^{2} h^{2}+a_{3} f \sigma^{3}+a_{4} h^{4} .
$$

In order to retrieve the formulae in a general gauge it suffices to replace $h^{2}$ by the $\mathrm{SO}(4)$ invariant combination $h^{2}+\vec{\pi}^{2}$.

The only strictly necessary soft breaking terms are $\alpha$ and $\beta$ as they need to be present to absorb divergences generated by one-loop Coleman-Weinberg contributions to the Lagrangian, as shown in appendix A; only those terms will be considered in what follows, ${ }^{2}$ a procedure already previously adopted in ref. [22]. The potential then reads

$$
V(h, \sigma)=\lambda\left(h^{2}+\sigma^{2}-f^{2}\right)^{2}+\alpha f^{3} \sigma-\beta f^{2} h^{2},
$$

resulting on a system depending on four parameters. The scalar quartic coupling $\lambda$ can be conventionally traded by the $\sigma$ mass, given by $m_{\sigma}^{2} \simeq 8 \lambda f^{2}$ for negligible $\alpha$ and $\beta$; the non-linear model would be recovered in the limit $m_{\sigma} \gg f$, that is $\lambda \gg 1$.

A consistent electroweak (EW) symmetry breaking requires both scalars $h, \sigma$ to acquire a non-vanishing vev, respectively dubbed as $v$ and $v_{\sigma}$ below, as for $v \neq 0$ the $\mathrm{SO}(4)$ global group and the EW group are spontaneously broken. Note that the vev of $h$ is identified with the electroweak scale since it can be related to the Fermi constant precisely as in the $\mathrm{SM}$, see section 2.2 below. For $\alpha, \beta \neq 0$ and assuming $v \neq 0$, it results

$$
v_{\sigma}^{2}=f^{2} \frac{\alpha^{2}}{4 \beta^{2}} \quad, \quad v^{2}=f^{2}\left(1-\frac{\alpha^{2}}{4 \beta^{2}}+\frac{\beta}{2 \lambda}\right)
$$

satisfying the condition

$$
v^{2}+v_{\sigma}^{2}=f^{2}(1+\beta / 2 \lambda),
$$

which indicates that the $\mathrm{SO}(5)$ vev is "renormalized" by the $\beta$ term in the potential. From eqs. (2.10) and (2.11) it follows that both $f^{2}>0$ and $f^{2}<0$ are in principle allowed, ${ }^{3}$ in appropriate regions of the parameters $(\alpha, \beta, \lambda)$. However, in the $\mathrm{SO}(5)$-invariant limit, for negative $f^{2}$ the minimum of the potential is at the origin and in consequence the symmetry is unbroken and there are no Goldstone bosons. The focus of this paper is instead set on the

\footnotetext{
${ }^{1}$ Here we choose to get rid of the $\sigma^{2}$ and $\sigma^{4}$ terms.

${ }^{2}$ Full renormalizability of the theory requires, in general, the presence of all gauge invariant operators of dimension equal to or smaller than four. At two or more loops, the renormalization procedure may thus require to include further symmetry breaking terms beyond those considered; we will assume that their finite contributions will be weighted by comparatively negligible coefficients and can be safely omitted in our analysys.

${ }^{3}$ For $f^{2}<0, \alpha$ would have to be purely imaginary because of hermiticity.
} 
interpretation of the Higgs particle as a PNGB, which requires $f^{2}>0$ as well as $|v|<\left|v_{\sigma}\right|$, the latter condition defining the region in parameter space continuously connected with the limiting case $v=0$ in which the Higgs particle becomes a true Goldstone boson. For $f^{2}>0$, the positivity of $v^{2}$ in eq. (2.10) and the $|v|<\left|v_{\sigma}\right|$ constraint lead respectively to the conditions ${ }^{4}$

$$
\begin{aligned}
\alpha^{2} & <4 \beta^{2}\left(1+\frac{\beta}{2 \lambda}\right), \\
2 \beta^{2}\left(1+\frac{\beta}{2 \lambda}\right) & <\alpha^{2},
\end{aligned}
$$

which for $|\beta| \ll \lambda$ would indicate $2 \beta^{2} \lesssim \alpha^{2} \lesssim 4 \beta^{2}$. Moreover, in order to get $v^{2} \ll f^{2}$, eq. (2.10) requires a fine-tuning such that $\alpha / 2 \beta$ is very close to unity.

Expanding the $\sigma$ and $h$ fields around their minima, $h \equiv \hat{h}+v$ and $\sigma \equiv \hat{\sigma}+v_{\sigma}$, and diagonalizing the scalar mass matrix, the mass eigenstates are given by

$$
h_{\text {phys }}=\hat{h} \cos \gamma-\hat{\sigma} \sin \gamma \quad, \quad \sigma_{\text {phys }}=\hat{\sigma} \cos \gamma+\hat{h} \sin \gamma .
$$

For simplicity, from now on the notation $h_{\text {phys }}$ and $\sigma_{\text {phys }}$ will be traded by $h$ and $\sigma$, respectively. The mixing angle in eq. (2.14) is given by

$$
\tan 2 \gamma=\frac{4 v v_{\sigma}}{3 v_{\sigma}^{2}-v^{2}-f^{2}}
$$

and should remain in the interval $\gamma \in[-\pi / 4, \pi / 4]$ in order not to interchange the roles of the heavy and light mass eigenstates. The mass eigenvalues are given by

$$
m_{\text {heavy, light }}^{2}=4 \lambda f^{2}\left\{\left(1+\frac{3}{4} \frac{\beta}{\lambda}\right) \pm\left[1+\frac{\beta}{2 \lambda}\left(1+\frac{\alpha^{2}}{2 \beta^{2}}+\frac{\beta}{8 \lambda}\right)\right]^{1 / 2}\right\},
$$

where the plus sign refers to the heavier eigenstate. For $f^{2}>0$, the squared masses are positive if the following two conditions are satisfied ${ }^{5}$

$$
3 \beta+4 \lambda>0, \quad 2 \beta^{2}+4 \beta \lambda-\alpha^{2} \lambda / \beta>0,
$$

with the second constraint coinciding with that in eq. (2.12) multiplying it by $1 /(4 \beta \lambda)$; it follows that $\beta>0$. If the soft mass term proportional to $\beta$ in the scalar potential eq. (2.9) would be overall positive (as for instance for $f^{2} j 0$ and $\beta>0$ ), the minimum would always correspond to an undesired symmetric EW vacuum $v=0$. Assuming the $\mathrm{SO}(5)$ explicit breaking to be small, $|\beta| / 4 \lambda \ll 1$ which may only happen for positive $f^{2}$, the masses of the heavy and light eigenstates read

$$
\begin{aligned}
& m_{\text {heavy }}^{2}=8 \lambda f^{2}+2 \beta\left(3 f^{2}-v^{2}\right)+O\left(\frac{\beta}{4 \lambda}\right), \\
& m_{\text {light }}^{2}=2 \beta v^{2}+O\left(\frac{\beta}{4 \lambda}\right) .
\end{aligned}
$$

\footnotetext{
${ }^{4}$ For $f^{2}<0$, the inequality eq. (2.12) is reverted.

${ }^{5}$ For $f^{2}<0$, both inequalities in eq. (2.17) are reverted and as a consequence $\beta<0$.
} 
The physical scalars thus correspond to a "light" state with mass $O(\sqrt{\beta} v)$ and a "heavy" state with mass $O(\sqrt{\lambda} f)$. It will be later shown that, for a PNGB Higgs particle (that is $v<v_{\sigma}$ and $f^{2}>0$ ), the less fine-tuned regions in parameter space correspond to the case $m_{\text {light }}=m_{h}$ and $m_{\text {heavy }}=m_{\sigma}$ in the equations above. In fact, would the $\sigma$ particle be lighter than the Higgs, the roles of the lighter and heavier eigenstates would be flipped and the mixing angle $\gamma$ will be necessarily outside the region quoted above. The lighter $\sigma$ scenario is quite different from the typical Higgs PNGB scenarios considered in the literature.

Notice that for $m_{h}<m_{\sigma}$ and at variance with the SM case, in the regime of small soft $\mathrm{SO}(5)$ breaking the mass of the Higgs and its quartic self-coupling are controlled by two different parameters, $\beta$ and $\lambda$, respectively. This is consistent with the PNGB nature of the Higgs boson whose mass should now appear protected from growing in the strong interacting regime of the theory - corresponding to large $\lambda$ - in which instead the $\sigma$ mass would increase. In other words, we have replaced the hierarchy problem for the Higgs particle mass by a sensitivity of the $\sigma$ particle to heavier scales: the $\sigma$ mass represents generically the heavy UV completion. The expression for $m_{h}$ shows that the value of the $\beta$ parameter for small $\beta / 4 \lambda$ is expected to be $\beta \sim m_{h}^{2} / 2 v^{2} \sim 0.13$.

\section{$2.2 \quad$ Scalar-gauge boson couplings}

In the unitary gauge, the kinetic scalar Lagrangian written in terms or the unrotated fields reads

$$
\mathcal{L}_{s, \text { kin }}=\frac{1}{2}\left(\partial_{\mu} \hat{\sigma}\right)^{2}+\frac{1}{2}\left(\partial_{\mu} \hat{h}\right)^{2}+\frac{g^{2}}{4}(\hat{h}+v)^{2} W_{\mu}^{+} W^{\mu-}+\frac{\left(g^{2}+g^{\prime 2}\right)}{8}(\hat{h}+v)^{2} Z_{\mu} Z^{\mu},
$$

and justifies the previous identification of the Higgs vev $v$ with the electroweak scale defined from the $W$ mass

$$
v=246 \mathrm{GeV} .
$$

The rotation to the physical $h, \sigma$ fields results in the following Lagrangian for the scalar and scalar-gauge interactions, for $m_{h}<m_{\sigma}$,

$$
\begin{aligned}
\mathcal{L}_{s}= & \frac{1}{2}\left(\partial_{\mu} \sigma\right)^{2}+\frac{1}{2}\left(\partial_{\mu} h\right)^{2}-\frac{1}{2} m_{\sigma}^{2} \sigma^{2}-\frac{1}{2} m_{h}^{2} h^{2}-\lambda\left(h^{2}+2 h \sigma+\sigma^{2}\right)^{2} \\
& -4 \lambda\left(v \cos \gamma-v_{\sigma} \sin \gamma\right)\left(h^{3}+h \sigma^{2}\right)-4 \lambda\left(v \sin \gamma+v_{\sigma} \cos \gamma\right)\left(\sigma^{3}+h^{2} \sigma\right) \\
& +\left(1+\frac{h}{v} \cos \gamma+\frac{\sigma}{v} \sin \gamma\right)^{2}\left(M_{W}^{2} W_{\mu}^{+} W^{\mu-}+\frac{1}{2} M_{Z}^{2} Z_{\mu} Z^{\mu}\right)
\end{aligned}
$$

The physical Higgs couplings are thus seen to be weighted down by a $\cos \gamma$ factor with respect to the SM Higgs ones, while the $\sigma$ field acquires the same interactions than $h$ albeit weighted down by $\sin \gamma$, a fact rich in phenomenological consequences to be discussed further below. The SM limit is recovered when the $\sigma$ field is decoupled from the spectrum and $\cos \gamma=1$ follows from eqs. (2.10) and (2.15). Conversely, for $m_{h}>m_{\sigma}$ the mixing dependence would correspond to the interchange $\cos \gamma \leftrightarrow \sin \gamma$ in eq. (2.20). 


\subsection{Renormalization and scalar tree-level decays}

The four independent parameters of the scalar Lagrangian can be expressed in terms of four observables, which we choose to be:

$$
G_{F} \equiv\left(\sqrt{2} v^{2}\right)^{-1}, \quad m_{h}, \quad m_{\sigma}, \quad \sin \gamma,
$$

with the Fermi constant $G_{F}$ as measured from muon decay and $m_{h}$ from the Higgs pole mass, while $m_{\sigma}$ could be determined from future measurements of the $\sigma$ mass, and $\sin \gamma$ from either deviations of the Higgs couplings or from the $\sigma$ line shape obtained from its decay into four leptons for $m_{\sigma} \geq 300 \mathrm{GeV}$, analogous to the case of a heavy SM Higgs boson. ${ }^{6}$

Using eqs. (2.10), (2.15), (2.16) and (2.19), the exact expressions for the $h$ and $\sigma$ vevs in terms of those physical parameters can be obtained

$$
\begin{aligned}
v & =\left(\sqrt{2} G_{F}\right)^{-1 / 2}, \\
v_{\sigma} & =\frac{v \sin (2 \gamma)\left(m_{\sigma}^{2}-m_{h}^{2}\right)}{m_{\sigma}^{2}+m_{h}^{2}-\left(m_{\sigma}^{2}-m_{h}^{2}\right) \cos (2 \gamma)} .
\end{aligned}
$$

These expressions in turn allow to express in terms of measured quantities the four independent parameters of the scalar potential eq. (2.9), which can be written as

$$
\begin{aligned}
\lambda & =\frac{\sin ^{2} \gamma m_{\sigma}^{2}}{8 v^{2}}\left(1+\cot ^{2} \gamma \frac{m_{h}^{2}}{m_{\sigma}^{2}}\right), \\
\frac{\beta}{4 \lambda} & =\frac{m_{h}^{2} m_{\sigma}^{2}}{\sin ^{2} \gamma m_{\sigma}^{4}+\cos ^{2} \gamma m_{h}^{4}-2 m_{h}^{2} m_{\sigma}^{2}}, \\
\frac{\alpha^{2}}{4 \beta^{2}} & =\frac{\sin ^{2}(2 \gamma)\left(m_{\sigma}^{2}-m_{h}^{2}\right)^{2}}{4\left(\sin ^{2} \gamma m_{\sigma}^{4}+\cos ^{2} \gamma m_{h}^{4}-2 m_{h}^{2} m_{\sigma}^{2}\right)}, \\
f^{2} & =\frac{v^{2}\left(\sin ^{2} \gamma m_{\sigma}^{4}+\cos ^{2} \gamma m_{h}^{4}-2 m_{h}^{2} m_{\sigma}^{2}\right)}{\left(\sin ^{2} \gamma m_{\sigma}^{2}+\cos ^{2} \gamma m_{h}^{2}\right)^{2}} .
\end{aligned}
$$

The above exact formulae show that the mixing angle $\gamma$ does not coincide with the parameter $v / f \equiv \sqrt{\xi}$ commonly used in the literature about composite Higgs models, except in the limit $m_{\sigma} \gg m_{h}$ (or more precisely $\beta / 4 \lambda \ll 1$ and $v^{2} \ll f^{2}$ ), where for sizeable sin $\gamma$ the last equation above leads to

$$
\sin ^{2} \gamma \underset{m_{\sigma} / m_{h} \gg 1}{\longrightarrow} \frac{v^{2}}{f^{2}}-4 \frac{m_{h}^{2}}{m_{\sigma}^{2}} .
$$

A few comments regarding the parameter space and the scalar spectrum are in order, as arbitrary values of $m_{\sigma}$ and $\sin ^{2} \gamma$ are not allowed if we insist on interpreting the Higgs

\footnotetext{
${ }^{6}$ For a lighter $\sigma$, the decay width becomes too narrow - possibly even below the experimental resolution - and more ingenious procedures would be required to determine the scalar mixing strength, such as for instance on-shell to off-shell cross section measurements [28].
} 


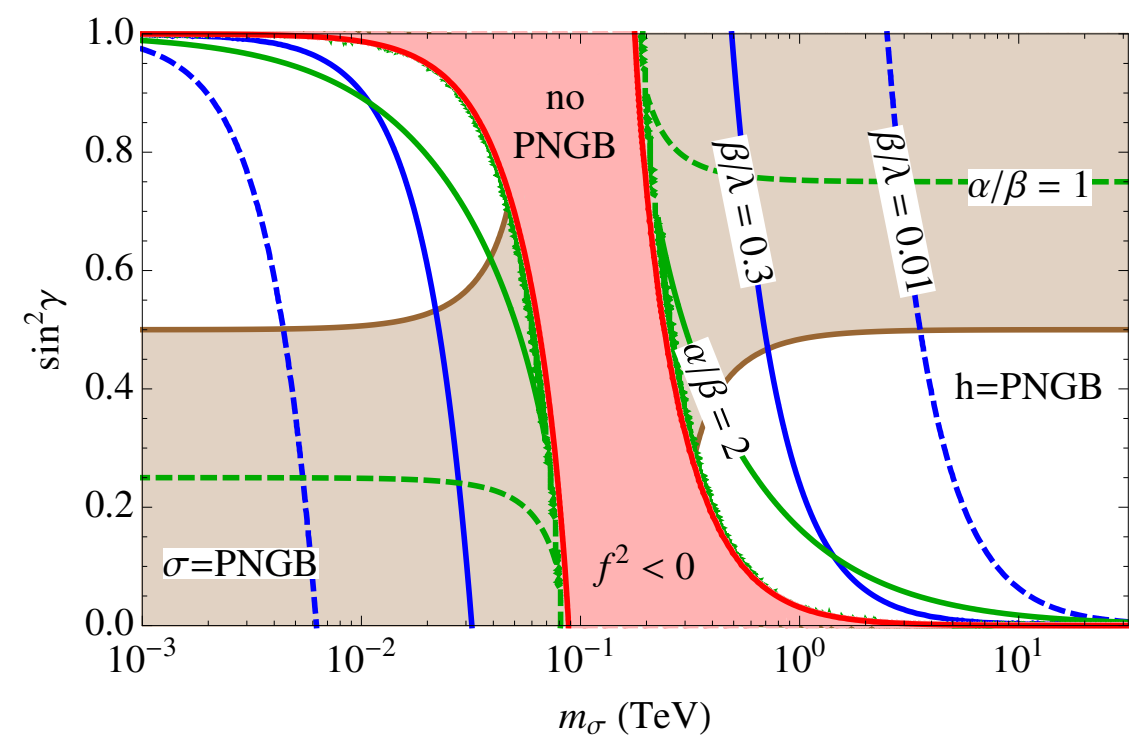

Figure 1. $m_{\sigma}$ versus $\sin ^{2} \gamma$ parameter space of the scalar sector. The Higgs mass $m_{h}$ and the Higgs vev $v$ have been fixed to their physical values. The red region corresponds to $f^{2}<0$, for which the $\mathrm{SO}(5)$-invariant part of the potential is unbroken and there are no Goldstone bosons in the symmetric limit. The region where $|v|>\left|v_{\sigma}\right|$ is shown in brown (these regions are excluded by Higgs data, see text). The Higgs is a pseudo-Goldstone boson within the white regions at the bottom-right and the top-left part of the plane.

boson as the pseudogoldstone boson of a spontaneous $\mathrm{SO}(5)$ breaking. Figure 1 displays the $\left(m_{\sigma}, \sin ^{2} \gamma\right)$ plane: at each point the scalar sector is completely defined as $m_{h}$ and $v$ are fixed to their physical values. The differently colored regions correspond to

- No $\mathrm{SO}(5)$ breaking in the light red region, where $f^{2}<0$; its red borders depict the $f^{2}=0$ frontier;

- The $\sigma$ particle being the PNGB of the spontaneous breaking of $\mathrm{SO}(5)$ in the light brown region, where $v_{\sigma}<v$;

- The Higgs as the PNGB of the $\mathrm{SO}(5) \rightarrow \mathrm{SO}(4)$ breaking in the white areas, where $v<v_{\sigma}$ and the Higgs would became a true goldstone boson in the absence of EW breaking $(v \rightarrow 0)$.

A complementary divide is provided by the value of the Higgs mass:

- On the $m_{h}<m_{\sigma}$ region to the right of the figure, the physical Higgs couplings to SM particles are weighted down by $\cos \gamma$ with respect to SM values, see for instance eq. (2.20). It will be shown in the next sections that present LHC Higgs data only allow for values $\sin ^{2} \gamma<0.18$ at $2 \sigma \mathrm{CL}$, though, leaving as allowed parameter space a fraction of the lower white (Higgs PNGB) section of the figure. The analysis in the next sections will thus focus in this regime, for which figure 1 already suggests a lower bound on $m_{\sigma}$ of a few hundreds of $\mathrm{GeV}$. The relative importance of the soft breaking terms is also illustrated through the curves depicted for fixed $\alpha / \beta$ and $\beta / \lambda$; 
- On the $m_{\sigma}<m_{h}$ area to the right of the figure, the physical Higgs couplings to SM particles are instead weighted down by $\sin \gamma$, whose value will thus be bounded by $\sin ^{2} \gamma>0.82$ at $2 \sigma \mathrm{CL}$. It thus remains as available zone the upper part of the upper white (Higgs PNGB) region. Nevertheless, the quartic coupling $\lambda$ is there very small, typically $\lambda<10^{-3}$, making the $\mathrm{SO}(5)$ invariant potential very flat and potentially unstable against radiative corrections; furthermore, if the soft breaking parameters are required to be small compared to the symmetric term, $\alpha, \beta<\lambda$, their values may require extra fine-tuning with respect to radiative corrections from the fermionic sector to be discussed further below. For these reasons we will not dwell further below on the case $m_{\sigma}<m_{h}$ even if phenomenologically of some interest.

Extending the renormalization scheme to the gauge sector, we choose the two extra observables needed to be the mass of the $Z$ boson and the fine structure constant,

$$
M_{Z}, \quad \alpha_{\mathrm{em}}=\frac{e^{2}}{4 \pi},
$$

with $M_{Z}$ and $\alpha_{\mathrm{em}}$ as determined from Z-pole mass measurements and from Thompson scattering, respectively [29]. In our model, the relation between the gauge boson masses is the same than that for the SM,

$$
M_{W}=\cos \theta_{W} M_{Z},
$$

where the weak angle is given at tree-level by

$$
\sin ^{2} \theta_{W}=\frac{1}{2}\left(1-\sqrt{1-\frac{4 \pi \alpha_{\mathrm{em}}}{\sqrt{2} G_{F} M_{Z}^{2}}}\right) .
$$

Using all the above, it is straightforward to compute the relevant tree-level branching ratios for the heavy and light scalar boson decays into SM bosons:

$$
\begin{aligned}
\Gamma\left(h \rightarrow W W^{*}\right) & =\Gamma_{\mathrm{SM}}\left(h \rightarrow W W^{*}\right) \cos ^{2} \gamma, \\
\Gamma\left(h \rightarrow Z Z^{*}\right) & =\Gamma_{\mathrm{SM}}\left(h \rightarrow Z Z^{*}\right) \cos ^{2} \gamma, \\
\Gamma\left(\sigma \rightarrow W^{+} W^{-}\right) & =\frac{\sqrt{2} G_{F}}{16 \pi} m_{\sigma}^{3} \sin ^{2} \gamma\left[1+\mathcal{O}\left(\frac{M_{W}^{2}}{m_{\sigma}^{2}}\right)\right], \\
\Gamma(\sigma \rightarrow Z Z) & =\frac{\sqrt{2} G_{F}}{32 \pi} m_{\sigma}^{3} \sin ^{2} \gamma\left[1+\mathcal{O}\left(\frac{M_{Z}^{2}}{m_{\sigma}^{2}}\right)\right], \\
\Gamma(\sigma \rightarrow h h) & =\frac{\sqrt{2} G_{F}}{32 \pi} m_{\sigma}^{3} \sin ^{2} \gamma\left[1+\mathcal{O}\left(\frac{m_{h}^{2}}{m_{\sigma}^{2}}\right)\right],
\end{aligned}
$$

where the SM widths can be found for instance in ref. [30]. The $\sigma$ partial widths above will dominate the total $\sigma$ width unless the mixing is unnaturally tiny, and thus measuring the branching ratios is not enough to infer the value of the scalar mixing. It is easy to see that

$$
\frac{\Gamma_{\sigma}}{m_{\sigma}} \simeq \frac{m_{\sigma}^{2} \sin ^{2} \gamma}{8 v^{2}}
$$




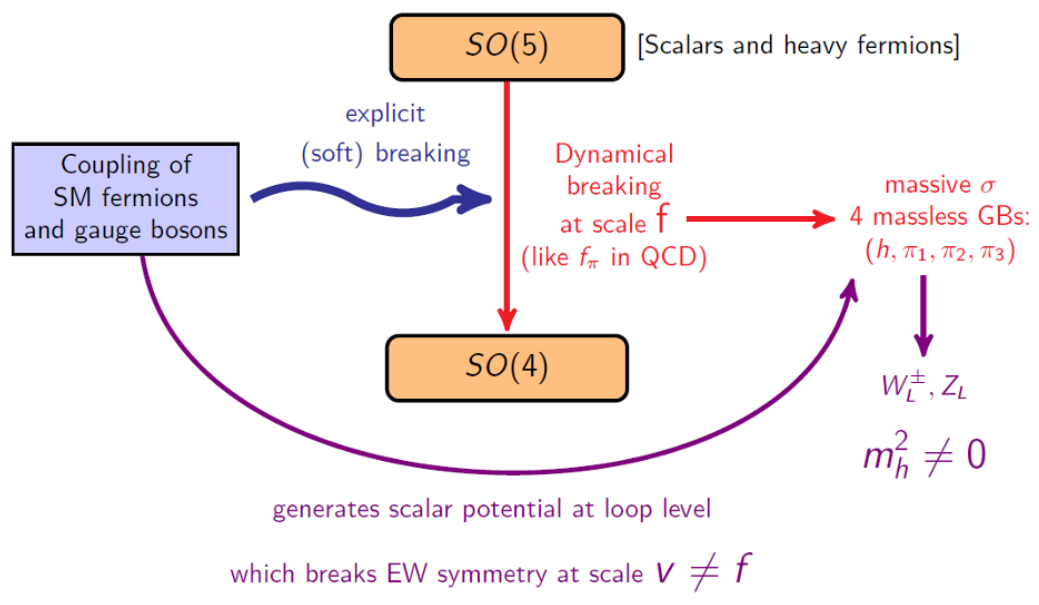

Figure 2. Schematics of the $\mathrm{SO}(5) \rightarrow \mathrm{SO}(4)$ model.

and thus the measurement of the line shape of the $\sigma$ seems feasible only for $m_{\sigma}$ above the EW breaking scale (assuming non-negligible mixing). In that regime, the value of $\sin \gamma$ can be inferred from the line shape and all other observables in eq. (2.28) can then be predicted in terms of the physical parameters defining our renormalization scheme. Other bosonic decay channels requiring one-loop amplitudes will be discussed later on.

\section{Fermionic sector}

The fermionic sector is unavoidably an important source of model dependency as diverse choices of $\mathrm{SO}(5)$ fermionic multiplets are possible. Moreover the achievement of the desired symmetry breaking pattern in "composite Higgs" models relies on the fermionic sector. A schematic picture is given in figure 2 considering a high energy global symmetry group $\mathrm{SO}(5)$ in the case under discussion:

- Heavy scalar and fermion representations of the high-energy global symmetry are considered. $\mathrm{SO}(5)$ breaks spontaneously to $\mathrm{SO}(4)$ at a scale $f$, resulting in four massless Goldstone bosons: the three longitudinal components of the electroweak gauge bosons and a "Higgs Goldstone boson". The fifth component $\sigma$ remains massive.

- Furthermore, $\mathrm{SO}(5)$ is explicitly broken by the coupling of the exotic heavy representations to the SM fermions (soft breaking) and to the gauge bosons (hard breaking). This induces at one-loop a potential for the $h$ field with a non-trivial minimum, providing a mass for $h$ and breaking the SM electroweak symmetry at a scale $v \neq f$.

Several "minimal" possibilities have been explored in the literature for the exotic fermionic representations (see for instance refs. [15, 22]). The setup considered in this paper contains:

1. Heavy (exotic) vector-like fermions in complete representations of $\mathrm{SO}(5)$, either in the fundamental representation, denoted below by $\psi$ - or singlets denoted by $\chi$. 


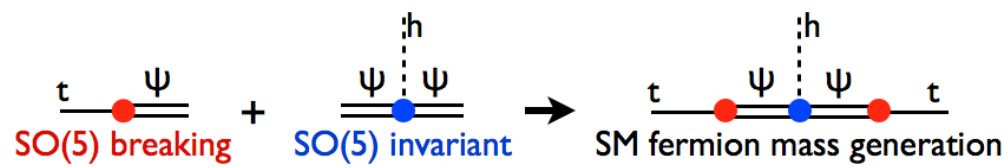

Figure 3. Schematics of light fermion mass generation. The light SM fermions - here the top quark - couple to the heavy partners breaking explicitly SO(5). The middle image depicts the $\mathrm{SO}(5)$ invariant Yukawa interactions between the Higgs and the heavy partners. The combination of both couplings induces and effective top Yukawa coupling and thus a massive top quark.

2. A scalar field $\phi$ in the fundamental representation of $\mathrm{SO}(5)$, which contains the $h$ and $\sigma$ particles. Its vev breaks $\mathrm{SO}(5)$ spontaneously to $\mathrm{SO}(4)^{\prime}$. By construction only the heavy exotic fermions couple directly to the scalar $\phi$.

3. The Higgs field couples to the exotic fermions only via $\mathrm{SO}(5)$ invariant Yukawa couplings. The sources of $\mathrm{SO}(5)$ breaking lie instead in the electroweak gauge interactions and in mixing terms between the heavy exotic fermions and the SM fermions. Such a breaking is fed via loop corrections to the scalar potential, where it is modeled by two $\mathrm{SO}(5)$ soft breaking terms which are custodial preserving.

This choice of fermionic representation respects an approximate custodial symmetry which protects the $Z b b$ coupling [31]. Figure 3 illustrates an intriguing characteristic of the fermionic sector in this class of models - which are often denominated by the generic name of "partial compositeness" [11]: a seesaw-like mechanism is at work in the generation of all low-energy fermion masses. The heavier the exotic fermions the lighter the light fermions.

To ensure correct hypercharge assignments for the SM fermions coupled directly to heavy exotic fields, the global symmetry is customarily enlarged by (at least) an extra $\mathrm{U}(1)_{X}$ sector, leading finally to a pattern of spontaneous global symmetry breaking given by

$$
\mathrm{SO}(5) \times \mathrm{U}(1)_{X} \rightarrow \mathrm{SO}(4) \times \mathrm{U}(1)_{X} \approx \mathrm{SU}(2)_{L} \times \mathrm{SU}(2)_{R} \times \mathrm{U}(1)_{X}
$$

with the hypercharge corresponding now to a combination of the new generator and that of $\mathrm{SU}(2)_{R}$ generator, see eq. (2.4),

$$
Y=\Sigma_{R}^{(3)}+X
$$

As the global $\mathrm{U}(1)_{X}$ symmetry remains unbroken, no additional Goldstone bosons are generated. Two different $\mathrm{U}(1)_{X}$ charges are compatible with SM hypercharge assignments: $2 / 3$ and $-1 / 3$. We will indeed consider two different copies of heavy fermions for each representation, differentiated by the $\mathrm{U}(1)_{X}$, as they are necessary to induce mass terms for both the SM up and the down quark sectors. Schematically, the fundamental and singlet representations can be decomposed under $\mathrm{SU}(2)_{L}$ quantum numbers as follows,

$$
\begin{aligned}
\psi^{(2 / 3)} & \sim\left(X, Q, T^{(5)}\right), & \psi^{(-1 / 3)} & \sim\left(Q^{\prime}, X^{\prime}, B^{(5)}\right), \\
\chi^{(2 / 3)} & \sim T^{(1)}, & \chi^{(-1 / 3)} & \sim B^{(1)},
\end{aligned}
$$




\begin{tabular}{|c|c|c|c|c|c|c|}
\hline Charge/Field & $X$ & $Q$ & $T_{(1,5)}$ & $Q^{\prime}$ & $X^{\prime}$ & $B_{(1,5)}$ \\
\hline$\Sigma_{R}^{(3)}$ & $+1 / 2$ & $-1 / 2$ & 0 & $+1 / 2$ & $-1 / 2$ & 0 \\
\hline $\mathrm{SU}(2)_{L} \times \mathrm{U}(1)_{Y}$ & $(2,+7 / 6)$ & $(2,+1 / 6)$ & $(1,+2 / 3)$ & $(2,+1 / 6)$ & $(2,-5 / 6)$ & $(1,-1 / 3)$ \\
\hline$x$ & $+2 / 3$ & $+2 / 3$ & $+2 / 3$ & $-1 / 3$ & $-1 / 3$ & $-1 / 3$ \\
\hline \multirow{2}{*}{$q_{\mathrm{EM}}$} & $X^{u}=+5 / 3$ & $Q^{u}=+2 / 3$ & $+2 / 3$ & $Q^{\prime u}=+2 / 3$ & $X^{\prime u}=-1 / 3$ & $-1 / 3$ \\
& $X^{d}=+2 / 3$ & $Q^{d}=-1 / 3$ & & $Q^{\prime d}=-1 / 3$ & $X^{\prime d}=-4 / 3$ & \\
\hline
\end{tabular}

Table 1. Heavy fermion charges assignments.

where $X^{\left({ }^{\prime}\right)}, Q^{\left({ }^{\prime}\right)}$ denote the two different $\mathrm{SU}(2)_{L}$ doublets contained in the fundamental representation of $\mathrm{SO}(5)$. In each multiplet, the first doublet has $\Sigma_{R}^{(3)}=1 / 2$ while the second one has $\Sigma_{R}^{(3)}=-1 / 2 . T_{(1,5)}, B_{(1,5)}$ denote instead $\mathrm{SU}(2)_{L} \times \mathrm{SU}(2)_{R}$ singlets, respectively in the 5 and 1 representation of $\mathrm{SO}(5)$. Table 1 summarizes the relevant quantum numbers for all heavy fermions.

The fermionic Lagrangian. For the SM fermions, the analysis below will be restricted to the third generation of SM quarks for simplicity, denoting by $q_{L}$ and $t_{R}$ and $b_{R}$ the doublet and singlets, respectively. It would be straightforward to extend the results to the other two generations, for instance introducing heavier replica of the exotic sector, leading to very minor additional phenomenological impact.

Assuming the "minimal" content specified in the previous sections, the fermionic Lagrangian is given by

$$
\begin{aligned}
\mathcal{L}_{F}= & \bar{q}_{L} i \not D q_{L}+\bar{t}_{R} i \not D t_{R}+\bar{b}_{R} i \not D b_{R} \\
+ & \bar{\psi}^{(2 / 3)}\left(i \not D-M_{5}\right) \psi^{(2 / 3)}+\bar{\psi}^{(-1 / 3)}\left(i \not D-M_{5}^{\prime}\right) \psi^{(-1 / 3)} \\
+ & \bar{\chi}^{(2 / 3)}\left(i \not D-M_{1}\right) \chi^{(2 / 3)}+\bar{\chi}^{(-1 / 3)}\left(i \not D-M_{1}^{\prime}\right) \chi^{(-1 / 3)} \\
- & {\left[y_{1} \bar{\psi}_{L}^{(2 / 3)} \phi \chi_{R}^{(2 / 3)}+y_{2} \bar{\psi}_{R}^{(2 / 3)} \phi \chi_{L}^{(2 / 3)}+y_{1}^{\prime} \bar{\psi}_{L}^{(-1 / 3)} \phi \chi_{R}^{(-1 / 3)}+y_{2}^{\prime} \bar{\psi}_{R}^{(-1 / 3)} \phi \chi_{L}^{(-1 / 3)}\right.} \\
& +\Lambda_{1}\left(\bar{q}_{L} \Delta_{2 \times 5}^{(2 / 3)}\right) \psi_{R}^{(2 / 3)}+\Lambda_{2} \bar{\psi}_{L}^{(2 / 3)}\left(\Delta_{5 \times 1}^{(2 / 3)} t_{R}\right)+\Lambda_{3} \bar{\chi}_{L}^{(2 / 3)} t_{R} \\
& \left.+\Lambda_{1}^{\prime}\left(\bar{q}_{L} \Delta_{2 \times 5}^{(-1 / 3)}\right) \psi_{R}^{(-1 / 3)}+\Lambda_{2}^{\prime} \bar{\psi}_{L}^{(-1 / 3)}\left(\Delta_{5 \times 1}^{(-1 / 3)} b_{R}\right)+\Lambda_{3}^{\prime} \bar{\chi}_{L}^{(-1 / 3)} b_{R}+\text { h.c. }\right] .
\end{aligned}
$$

The first lines contain the kinetic terms for the SM fermions. The second and third lines include the kinetic and mass terms for the exotic fermions. The kinetic terms become $\mathrm{SO}(5)$-invariant in the gaugeless limit. The fourth line contains the $\mathrm{SO}(5)$ invariant Yukawa couplings of the exotic sector to the Higgs field. Finally, the last two lines of the Lagrangian contain the $\mathrm{SO}(5)$ soft-breaking interactions of SM fermions with exotic fermions. $\Delta_{2 \times 5}$ and $\Delta_{5 \times 1}$ denote suitable spurions connecting $\mathrm{SO}(5)$ and $\mathrm{SU}(2) \times \mathrm{U}(1)$ representations. If the primed parameters were set to zero no bottom mass would be generated through this mechanism. All parameters in eq. (3.3) are assumed real for simplicity, that is, we will assume CP invariance in what follows. 
It is useful to rewrite the Lagrangian in eq. (3.3) in terms of $\mathrm{SU}(2)_{L}$ components. For this purpose, from this point and until eq. (3.12) below, $h$ and $\sigma$ will denote again the unshifted and unrotated original scalar fields in eq. (2.9):

$$
\begin{aligned}
\mathcal{L}_{F}= & \bar{q}_{L} i \not D q_{L}+\bar{t}_{R} i \not D t_{R}+\bar{b}_{R} i \not D b_{R}+\bar{Q}\left(i \not D-M_{5}\right) Q+\bar{X}\left(i \not D-M_{5}\right) X \\
+ & \bar{T}^{(5)}\left(i \not D-M_{5}\right) T^{(5)}+\bar{T}^{(1)}\left(i \not D-M_{1}\right) T^{(1)}+\bar{Q}^{\prime}\left(i \not D-M_{5}^{\prime}\right) Q^{\prime}+\bar{X}^{\prime}\left(i \not D-M_{5}^{\prime}\right) X^{\prime} \\
+ & \bar{B}^{(5)}\left(i \not D-M_{5}^{\prime}\right) B^{(5)}+\bar{B}^{(1)}\left(i \not D-M_{1}^{\prime}\right) B^{(1)} \\
- & {\left[y_{1}\left(\bar{X}_{L} H T_{R}^{(1)}+\bar{Q}_{L} \widetilde{H} T_{R}^{(1)}+\bar{T}_{L}^{(5)} \sigma T_{R}^{(1)}\right)+y_{2}\left(\bar{T}_{L}^{(1)} H^{\dagger} X_{R}+\bar{T}_{L}^{(1)} \widetilde{H}^{\dagger} Q_{R}+\bar{T}_{L}^{(1)} \sigma T_{R}^{(5)}\right)\right.} \\
& +y_{1}^{\prime}\left(\bar{X}^{\prime}{ }_{L} \widetilde{H} B_{R}^{(1)}+\bar{Q}^{\prime}{ }_{L} H B_{R}^{(1)}+\bar{B}_{L}^{(5)} \sigma B_{R}^{(1)}\right)+y_{2}^{\prime}\left(\bar{B}_{L}^{(1)} \widetilde{H}^{\dagger} X_{R}^{\prime}+\bar{B}_{L}^{(1)} H^{\dagger} Q_{R}^{\prime}+\bar{B}_{L}^{(1)} \sigma B_{R}^{(5)}\right) \\
& \left.+\Lambda_{1} \bar{q}_{L} Q_{R}+\Lambda_{1}^{\prime} \bar{q}_{L} Q_{R}^{\prime}+\Lambda_{2} \bar{T}_{L}^{(5)} t_{R}+\Lambda_{3} \bar{T}_{L}^{(1)} t_{R}+\Lambda_{2}^{\prime} \bar{B}_{L}^{(5)} b_{R}+\Lambda_{3}^{\prime} \bar{B}_{L}^{(1)} b_{R}+\text { h.c. }\right] .
\end{aligned}
$$

Eq. (3.4) shows that the light fermion masses must be proportional to the $\mathrm{SO}(5)$ invariant Yukawa couplings of heavy fermions and to the explicitly $\mathrm{SO}(5)$ breaking light-heavy fermionic interactions. The generation of light quark masses requires a vev for the scalar doublet $H$. For instance, a $\bar{t}_{L} t_{R}$ mass term is seen to result from the following chain of couplings,

$$
q_{l} \underset{\Lambda_{1}}{\longrightarrow} Q_{R} \underset{M_{5}}{\longrightarrow} Q_{L} \underset{y_{1}\langle\tilde{H}\rangle}{\longrightarrow} T_{R}^{(1)} \underset{M_{1}}{\longrightarrow} T_{L}^{(1)} \underset{\Lambda_{3}}{\longrightarrow} t_{R}
$$

suggesting

$$
m_{t} \propto y_{1} \frac{\Lambda_{1} \Lambda_{3}}{M_{1} M_{5}} v
$$

see also figure 3 and section 6 . Furthermore, both the $+2 / 3$ and $-1 / 3$ electrically charged sectors acquire off-diagonal mixing terms.

The expression for the fermionic Lagrangian eq. (3.4) can be rewritten in a compact form defining a fermionic vector whose components are ordered by their electrical charges $q_{\mathrm{EM}}=(+5 / 3,+2 / 3,-1 / 3,-4 / 3)$,

$$
\Psi=\left(X^{u}, \mathcal{T}, \mathcal{B}, X^{\prime d}\right)
$$

where $\mathcal{T}$ and $\mathcal{B}$ include the top and bottom quarks together with their heavy fermionic partners

$$
\mathcal{T}=\left(t, Q^{u}, X^{d}, T^{(5)}, T^{(1)}, Q^{\prime u}\right), \quad \mathcal{B}=\left(b, Q^{\prime d}, X^{\prime u}, B^{(5)}, B^{(1)}, Q^{d}\right)
$$

The fermion mass terms in the weak basis can then be written as

$$
\mathcal{L}_{\mathcal{M}}=-\bar{\Psi}_{L} \mathcal{M}(h, \sigma) \Psi_{R}
$$


where here and in what follows the sum over all components of the fermionic vector is left implicit and the block diagonal $14 \times 14$ fermion mass matrix $\mathcal{M}$ reads

$$
\begin{aligned}
\mathcal{M}(h, \sigma) & =\operatorname{diag}\left(M_{5}, \mathcal{M}^{\mathcal{T}}(h, \sigma), \mathcal{M}^{\mathcal{B}}(h, \sigma), M_{5}^{\prime}\right), \\
\mathcal{M}^{\mathcal{T}}(h, \sigma) & =\left(\begin{array}{cccccc}
0 & \Lambda_{1} & 0 & 0 & 0 & \Lambda_{1}^{\prime} \\
0 & M_{5} & 0 & 0 & y_{1} \frac{h}{\sqrt{2}} & 0 \\
0 & 0 & M_{5} & 0 & y_{1} \frac{h}{\sqrt{2}} & 0 \\
\Lambda_{2} & 0 & 0 & M_{5} & y_{1} \sigma & 0 \\
\Lambda_{3} & y_{2} \frac{h}{\sqrt{2}} & y_{2} \frac{h}{\sqrt{2}} & y_{2} \sigma & M_{1} & 0 \\
0 & 0 & 0 & 0 & 0 & M_{5}^{\prime}
\end{array}\right), \\
\mathcal{M}^{\mathcal{B}}(h, \sigma) & =\mathcal{M}^{\mathcal{T}}(h, \sigma) \text { with }\left\{y_{i}, \Lambda_{i}, M_{i}\right\} \leftrightarrow\left\{y_{i}^{\prime}, \Lambda_{i}^{\prime}, M_{i}^{\prime}\right\} .
\end{aligned}
$$

The mass matrices can be diagonalised by bi-unitary (or for the case of the real parameters bi-orthogonal) transformations,

$$
\Psi_{L}^{\text {phys }}=L \Psi_{L}, \quad \Psi_{R}^{\text {phys }}=R \Psi_{R}, \quad \mathcal{M}^{\text {diag }}=L^{\dagger} \mathcal{M} R .
$$

These matrices can be diagonalized analytically in some interesting limits; in general they will be diagonalized numerically.

The physical light eigenstates are admixtures of the light and heavy fermion fields appearing in the Lagrangian. The scalar fields vevs induce in addition heavy fermion mass splittings. Notice however that, even in the limit of vanishing Yukava couplings, the exotic fermions get mixed via the $\mathrm{SO}(5)$ breaking couplings. Moreover, although the various dimensional couplings $\Lambda_{i}$ and $\Lambda_{i}^{\prime}$ in eqs. (3.3) and (3.4) may be of the same order, the top and bottom components of the heavy doublets are splitted by $\mathrm{SO}(4)$ breaking terms, generically of $O\left(y_{i} v\right)$.

\section{Phenomenology}

In this section, bounds are derived first on the model parameters resulting from present LHC Higgs data and from electroweak precision tests - namely $S, T$ and $g_{L}^{b}$. Future signals are discussed next, focusing in particular on $\sigma$ physics.

\subsection{Bounds from Higgs measurements}

The tree-level mixing of the scalar singlet $\sigma$ with the Higgs resonance $h$ can be strongly bounded from present data and in particular from $h$ to $Z Z$ and $W^{+} W^{-}$decays, and from $h$-gluon-gluon transitions: the Higgs coupling strength to SM fields is weighted down simply by a $\cos \gamma$ factor with respect the SM value, as previously explained and shown in eq. (2.20). We use the latest ATLAS and CMS combined results for the gluon-gluon and vector boson mediated Higgs production processes [32].

A $\chi^{2}$ fit taking into account the correlation between the corresponding coupling modifiers in the combined fit of the 7 and $8 \mathrm{TeV}$ LHC data — given by figure 23.B of ref. [32] 
— constrains directly $\cos \gamma$, translating into the following bound

$$
\sin ^{2} \gamma \lesssim 0.18(\text { at } 2 \sigma)
$$

which in the $m_{\sigma} \gg m_{h}$ limit would point to a value for the non-linearity parameter of composite Higgs models, $\xi \equiv v^{2} / f^{2} \sim \sin ^{2} \gamma$, consistent with the limits found in the literature [33], see eqs. (2.23) and (2.24) and the discussion below.

Comparison with literature on non-linear realizations. Ref. [33] shows that in non-linear realizations of the composite Higgs scenario the behaviour of the Higgs couplings modifications varies depending on the $\mathrm{SO}(5)$ fermionic representations chosen. In particular they compare the so called $\mathrm{MCHM}_{4}$ and $M C H M_{5}$ scenarios:

- In $\mathrm{MCHM}_{4}$, the fermions (both the embedded light ones and the heavy partners) are in the 4 (spinorial) representation of $\mathrm{SO}(5)$; the coupling modifiers then obey $k_{V}^{(4)}=k_{F}^{(4)}=(1-\xi)^{1 / 2}$, leading to a bound from Higgs data $\xi^{(4)}<0.18$ at $2 \sigma$ CL. $\mathrm{MCHM}_{4}$ is actually ruled out by its impact on the $\mathrm{Zbb}$ coupling and thus for instance disregarded in ref. [15].

- In $\mathrm{MCHM}_{5}$, the fermions are instead in the $\mathbf{5}$ (fundamental) of $\mathrm{SO}(5)$, and in this case $k_{V}^{(5)}=(1-\xi)^{1 / 2}$ differs from $k_{F}^{(5)}=(1-2 \xi)(1-\xi)^{-1 / 2}$, that is, $k_{F}^{(5)} / k_{V}^{(5)} \approx 1-\xi$ for small $\xi$ values. LHC Higgs data set then a bound $\xi^{(5)}<0.12$ at $2 \sigma$ CL.

Now, the heavy fermion configuration of our model is different from both scenarios, due to the role of $T^{(1)}$, a $\mathbf{1}$ (singlet) of $\mathrm{SO}(5)$, which mixes with $t_{R}$. According to the notation in ref. [15], the fermion representation in our model would be given by $M C H M_{5-5,1-5,1}$. Indeed, the effective top Yukawa requires only one Higgs insertion, which in the non-linear regime reads $y_{t} f \bar{t}_{L} t_{R} \sin (h / f)$, leading to $k_{F}=\sqrt{1-\xi}$. This is the same modification to the Higgs couplings as in $\mathrm{MCHM}_{4}$.

\subsection{Precision electroweak constraints}

Analyses available on precision tests for composite Higgs models, such as that in ref. [35], tend to consider non-linear versions of the theory where the only scalar present is the Higgs particle, but for ref. [22], which discusses qualitatively the interplay of scalar and exotic fermion contributions. see also ref. [24]. We present here an explicit computation of the scalar ( $h$ and $\sigma$ ) and exotic fermion contributions, discussing the impact of varying the $\sigma$ mass. $S, T$ and $U$ parameters are considered together with $g_{L}^{b}$ and parameter correlations.

\subsection{1 $S, T$ and $g_{L}^{b}$}

Consider the parameter definitions in ref. [36],

$$
\begin{aligned}
& \alpha S=\left.4 s_{W} c_{W} \frac{d \Pi_{30}\left(q^{2}\right)}{d q^{2}}\right|_{q^{2}=0}=4 s_{W} c_{W} F_{30}, \\
& \alpha T=\frac{1}{M_{W}^{2}}\left[\Pi_{11}(0)-\Pi_{33}(0)\right]=\frac{1}{M_{W}^{2}}\left[A_{11}-A_{33}\right], \\
& \alpha U=-\left.4 s_{W}^{2} \frac{d}{d q^{2}}\left[\Pi_{33}\left(q^{2}\right)-\Pi_{11}\left(q^{2}\right)\right]\right|_{q^{2}=0}=4 s_{W}^{2}\left(F_{11}-F_{33}\right),
\end{aligned}
$$


where $c_{W}\left(s_{W}\right)$ denotes the cosinus (sinus) of the Weinberg angle $c_{W}=M_{W} / M_{Z}$, and the electroweak vacuum polarization functions are given by

$$
\Pi_{i j}^{\mu \nu}(q)=-i\left[\Pi_{i j}\left(q^{2}\right) g^{\mu \nu}+\left(q^{\mu} q^{\nu}-\text { terms }\right)\right] ; \quad \Pi_{i j}\left(q^{2}\right) \equiv A_{i j}(0)+q^{2} F_{i j}+\ldots
$$

with $i, j=W, Z$ or $i, j=0,3$ for the $B$ or the $W_{3}$ bosons, respectively, and the dots indicating an expansion in powers of $q^{2}$. We will not consider further $U$ as it typically corresponds to higher order (mass dimension eight) couplings while only low-energy data (e.g. LEP) will be used here. ${ }^{7}$ On the contrary, relevant constraints could stem from deviations induced in the $Z \bar{b}_{L} b_{L}$ coupling, parametrized by $g_{L}^{b}$ in the decay amplitude

$$
\mathcal{M}_{Z \rightarrow \bar{b}_{L} b_{L}}=-\frac{e g_{L}^{b}}{s_{W} c_{W}} \bar{b}\left(p_{2}\right) \notin(q) \frac{1-\gamma_{5}}{2} b\left(p_{1}\right),
$$

where $\epsilon(q)$ denotes the $Z$ boson polarization and $p_{i}$ the $b$ quark and antiquark momenta.

The values of $S, T$ and $g_{L}^{b}$ are allowed to deviate from the SM prediction within the constraints $[35,38]$

$$
\begin{aligned}
\Delta S & \equiv S-S_{\mathrm{SM}}=0.0079 \pm 0.095 \\
\Delta T & \equiv T-T_{\mathrm{SM}}=0.084 \pm 0.062, \\
\Delta g_{L}^{b} & \equiv g_{L}^{b}-g_{L \mathrm{SM}}^{b}=(-0.13 \pm 0.61) \times 10^{-3},
\end{aligned}
$$

with the $\left(S, T, g_{L}^{b}\right)$ correlation matrix given by

$$
\left(\begin{array}{ccc}
1 & 0.864 & 0.06 \\
0.864 & 1 & 0.123 \\
0.06 & 0.123 & 1
\end{array}\right) \text {. }
$$

Scalar contributions in the linear $\mathrm{SO}(5)$ model: $h$ and $\sigma$. Given the scalar couplings in eq. (2.20), their contributions to $S$ and $T$ can be formulated as

$$
\begin{aligned}
& \Delta T^{(h \text { and } \sigma)}=-\Delta T_{\mathrm{SM}}^{h}\left(m_{h}\right)+c_{\gamma}^{2} \Delta T_{\mathrm{SM}}^{h}\left(m_{h}\right)+\Delta T^{(\sigma)}=s_{\gamma}^{2}\left[-\Delta T_{\mathrm{SM}}^{h}\left(m_{h}\right)+\Delta T_{\mathrm{SM}}^{h}\left(m_{\sigma}\right)\right] \\
& \Delta S^{(h \text { and } \sigma)}=-\Delta S_{\mathrm{SM}}^{h}\left(m_{h}\right)+c_{\gamma}^{2} \Delta S_{\mathrm{SM}}^{h}\left(m_{h}\right)+\Delta S^{(\sigma)}=s_{\gamma}^{2}\left[-\Delta S_{\mathrm{SM}}^{h}\left(m_{h}\right)+\Delta S_{\mathrm{SM}}^{h}\left(m_{\sigma}\right)\right]
\end{aligned}
$$

where the $\sigma$ contributions $\Delta T^{(\sigma)}$ and $\Delta S^{(\sigma)}$ have been simply written in terms of the usual $\mathrm{SM}$ formulae for the Higgs contribution $\Delta T_{\mathrm{SM}}^{h}$ and $\Delta S_{\mathrm{SM}}^{h}$ with the replacement $m_{h} \rightarrow m_{\sigma}$ and using the formulae valid for masses much above the electroweak scale. The scalar

\footnotetext{
${ }^{7}$ Some other handful parameter definitions are: $\epsilon_{1} \equiv \alpha T ; \epsilon_{3}=\alpha S /\left(4 s_{W}^{2}\right)$, see ref. [37].
} 
contribution to $\Delta T$ is then given by

$$
\begin{aligned}
\Delta T^{(h \text { and } \sigma)}=\frac{3 G_{F} M_{W}^{2}}{8 \pi^{2} \sqrt{2}} s_{\gamma}^{2}( & -m_{h}^{2} \frac{\log \left(m_{h}^{2} / M_{W}^{2}\right)}{M_{W}^{2}-m_{h}^{2}}+m_{\sigma}^{2} \frac{\log \left(m_{\sigma}^{2} / M_{W}^{2}\right)}{M_{W}^{2}-m_{\sigma}^{2}} \\
& \left.+\frac{M_{Z}^{2}}{M_{W}^{2}}\left\{m_{h}^{2} \frac{\log \left(m_{h}^{2} / M_{Z}^{2}\right)}{M_{Z}^{2}-m_{h}^{2}}-m_{\sigma}^{2} \frac{\log \left(m_{\sigma}^{2} / M_{Z}^{2}\right)}{M_{Z}^{2}-m_{\sigma}^{2}}\right\}\right),
\end{aligned}
$$

which in the limit $m_{\sigma} \gg m_{h}, M_{W}, M_{Z}$ reduces to

$$
\Delta T^{(h \text { and } \sigma)} \sim s_{\gamma}^{2} \frac{3 G_{F} M_{W}^{2}}{8 \pi^{2} \sqrt{2}} \frac{s_{W}^{2}}{c_{W}^{2}} \log \left(m_{\sigma}^{2} / M_{W}^{2}\right) .
$$

For the $\Delta S$ corrections, the formulation in refs. [39, 40] is used, leading to

$\alpha \Delta S_{\mathrm{SM}}^{h}(m)=s_{W}^{2} \frac{2 G_{F}}{\sqrt{2} \pi^{2}} M_{W}^{2}\left(\frac{x}{12(x-1)} \log (x)+\left(-\frac{x}{6}+\frac{x^{2}}{12}\right) F(x)-\left(1-\frac{x}{3}+\frac{x^{2}}{12}\right) F^{\prime}(x)\right)$,

where $x \equiv m^{2} / M_{Z}^{2}$ and for $x<4$ :

$$
\begin{aligned}
& F(x)=1+\left(\frac{x}{x-1}-\frac{1}{2} x\right) \log x-x \sqrt{\frac{4}{x}-1} \arctan \sqrt{\frac{4}{x}-1}, \\
& F^{\prime}(x)=-1+\frac{x-1}{2} \log x+(3-x) \sqrt{\frac{x}{4-x}} \arctan \sqrt{\frac{4}{x}-1},
\end{aligned}
$$

while for $x>4$ :

$$
\begin{aligned}
& F(x)=1+\left(\frac{x}{x-1}-\frac{1}{2} x\right) \log x-x \sqrt{1-\frac{4}{x}} \log \left(\sqrt{\frac{x}{4}-1}+\sqrt{\frac{x}{4}}\right), \\
& F^{\prime}(x)=-1+\frac{x-1}{2} \log x+(3-x) \sqrt{\frac{x}{x-4}} \log \left(\sqrt{\frac{x}{4}-1}+\sqrt{\frac{x}{4}}\right) .
\end{aligned}
$$

In the limit of very large $m_{\sigma}$, the $\sigma$ contribution to $S$ can be approximated by:

$$
\alpha \Delta S^{(\sigma)} \underset{\sigma \rightarrow \infty}{\longrightarrow} s_{\gamma}^{2} s_{W}^{2} \frac{2 G_{F}}{\sqrt{2} \pi^{2}} M_{W}^{2}\left[\frac{1}{12} \log \left(\frac{m_{\sigma}^{2}}{M_{W}^{2}}\right)\right]
$$

consistent with the statements in the literature for a very heavy Higgs particle [36].

The black curve in figure 4 displays examples of the $\Delta S$ and $\Delta T$ corrections induced by the $\sigma$ scalar as it follows from the formulae shown above. As earlier explained, the set of parameters in the scalar potential $(f, \lambda, \alpha, \beta)$ has been traded by four observables: $G_{F}$, $m_{h}, m_{\sigma}$ and the scalar mixing $\gamma$ (with the latter two yet to be experimentally measured). It is nevertheless theoretically illuminating to indicate the corresponding values for $f$ and the scalar quartic self-coupling $\lambda$ for each example analyzed, and their values are shown in all figures to follow. We present numerical results for two typical parameter regimes:

- $m_{\sigma}=2 \mathrm{TeV}, s_{\gamma}^{2}=0.04$, which corresponds to $f=1 \mathrm{TeV}$ and to scalar potential couplings $\lambda=0.38, \alpha=0.35$ and $\beta=0.16$, which clearly lie within the perturbative regime of the linear $\mathrm{SO}(5)$ sigma model. 


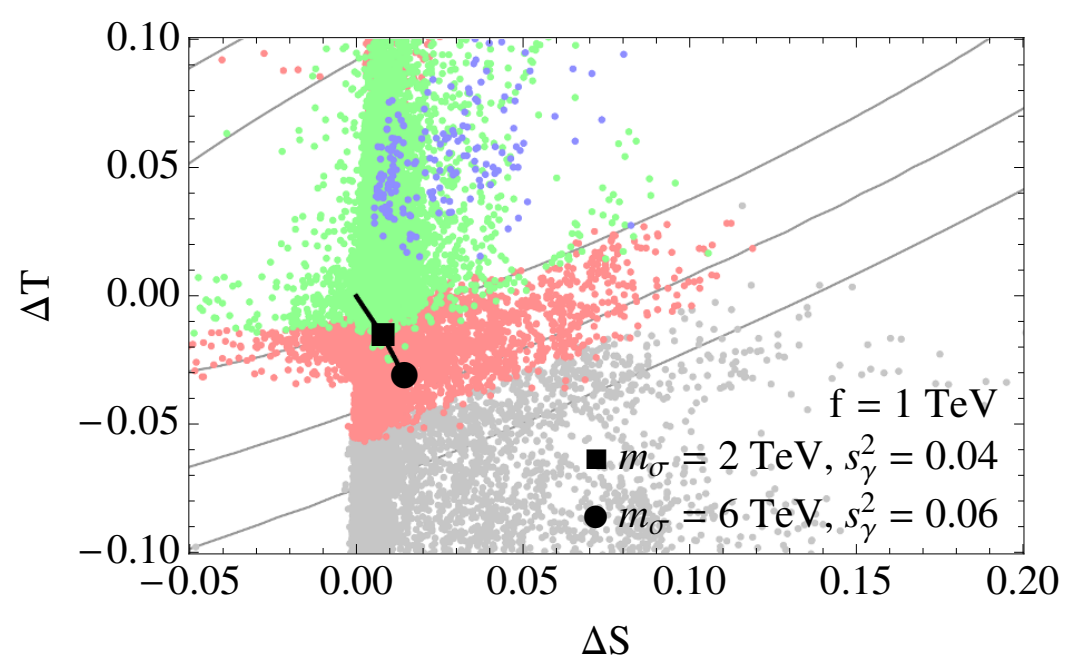

Figure 4. Uncombined contributions of the scalar sector (black curve) and the exotic fermionic sector to the parameters $S$ and $T$.

- $m_{\sigma}=6 \mathrm{TeV}, s_{\gamma}^{2}=0.06$, which also correspond to $f=1 \mathrm{TeV}$, while $\lambda=4.3$-closer to the limit of validity of the perturbative expansion- and $\alpha=0.25, \beta=0.13$; this pattern corresponds then to a mainly $\mathrm{SO}(5)$ symmetric scenario with small soft symmetry breaking.

Figure 4 shows a sizeable negative contribution of the $\sigma$ particle to $\Delta T$ which increases with $m_{\sigma}$, and positive contribution to $\Delta S$; the result is consistent with the pattern expected in ref. [22], and similar to that for the heavy Higgs case (see e.g. ref. [41]). In the limit $m_{\sigma} \rightarrow m_{h}$ the total scalar contribution matches that in the SM due to the Higgs particle. It is easy to extrapolate the $S$ and $T$ scalar contributions to other mixing regimes as they scale with $s_{\gamma}^{2}$ : for instance the effect would be amplified by a factor of $\sim 3$ when raising the mixing towards the maximal value allowed, see eq. (4.1).

For $g_{L}^{b}$ instead we will not analyze the one-loop $\sigma$ contributions, as they would be proportional to the bottom Yukawa couplings and thus negligible compared to the top and top-partner contributions to be discussed next.

Fermionic contributions. The heavy fermion sector may have an impact on the oblique parameters and on $g_{L}^{b}$. This sector adds additional parameter dependence on top of the four renormalization parameters already discussed for the scalar sector of the linear $\mathrm{SO}(5)$ sigma model. The fermionic parameter space is quite large and adjustable, and thus in practice $m_{\sigma}$ and $\gamma$ will be treated here as independent from them. It will also be assumed that the inclusion of quarks and leptons from the first two generations does not alter significantly the analysis of electroweak precision tests, as lighter fermions tend to have very small mixing with their heavy partners.

The gauge boson couplings to neutral (NC) and charged (CC) fermionic currents in the weak basis can be read from table 1 . After rotation to the mass basis, the corresponding 
Lagrangians can be written as [34]:

$$
\begin{aligned}
\mathcal{L}_{\mathrm{NC}} & =\bar{\Psi}^{\mathrm{phys}} \gamma^{\mu}\left[\frac{g}{2}\left(C_{L} P_{L}+C_{R} P_{R}\right) W_{\mu}^{3}-g^{\prime}\left(Y_{L} P_{L}+Y_{R} P_{R}\right) B_{\mu}\right] \Psi^{\mathrm{phys}} \\
& =\bar{\Psi}^{\mathrm{phys}} \gamma^{\mu}\left[\frac{g}{2 c_{W}}\left(C_{L} P_{L}+C_{R} P_{R}-2 s_{W}^{2} \mathcal{Q}\right) Z_{\mu}-e \mathcal{Q} A_{\mu}\right] \Psi^{\mathrm{phys}} \\
\mathcal{L}_{\mathrm{CC}} & =\bar{\Psi}^{\mathrm{phys}} \gamma^{\mu}\left[\frac{g}{\sqrt{2}}\left(V_{L} P_{L}+V_{R} P_{R}\right) W_{\mu}^{+}\right] \Psi^{\text {phys }}+\text { h.c. },
\end{aligned}
$$

where $P_{L}$ and $P_{R}$ are chirality projectors, $\Psi^{\text {phys }}$ denotes the generic fermionic vector in the physical mass basis and $e$ is the absolute value of the electric charge unit. In the model under discussion, the matrices $C$ and $Y$ are related via the electric charge matrix - see also eq. (3.2):

$$
Y_{\alpha}=\mathcal{Q}-\frac{1}{2} C_{\alpha} \quad \alpha=L \text { or } R
$$

with

$$
\mathcal{Q}=\left(+\frac{5}{3},+\frac{2}{3} \mathbf{1}_{6 \times 6},-\frac{1}{3} \mathbf{1}_{6 \times 6},-\frac{4}{3}\right)
$$

The relation between the NC coupling matrices in the mass basis, $C_{L, R}$ and $Y_{L, R}$, and their counterparts in the interaction basis (same symbols in curly characters below) is given by

$$
\begin{aligned}
C_{L} & =L \mathcal{C}_{L} L^{\dagger}, \quad C_{R}=R \mathcal{C}_{R} R^{\dagger}, \quad \mathcal{C}_{L ; R}=\operatorname{diag}\left(+1, \mathcal{C}_{L ; R}^{\mathcal{T}}, \mathcal{C}_{L ; R}^{\mathcal{B}},-1\right) \\
\mathcal{C}_{L ; R}^{\mathcal{T}} & =-\mathcal{C}_{L ; R}^{\mathcal{B}}=\operatorname{diag}(+1 ; 0,+1,-1,0,0,+1) ; \\
Y_{L} & =L \mathcal{Y}_{L} L^{\dagger}, \quad Y_{R}=R \mathcal{Y}_{R} R^{\dagger}, \quad \mathcal{Y}_{L ; R}=\operatorname{diag}\left(+\frac{7}{6}, \mathcal{Y}_{L ; R}^{\mathcal{T}}, \mathcal{Y}_{L ; R}^{\mathcal{B}},-\frac{5}{6}\right), \\
\mathcal{Y}_{L ; R}^{\mathcal{T}} & =\operatorname{diag}\left(\frac{1}{6} ; \frac{2}{3}, \frac{1}{6}, \frac{7}{6}, \frac{2}{3}, \frac{2}{3}, \frac{1}{6}\right), \quad \mathcal{Y}_{L ; R}^{\mathcal{B}}=\operatorname{diag}\left(\frac{1}{6} ;-\frac{1}{3}, \frac{1}{6},-\frac{5}{6},-\frac{1}{3},-\frac{1}{3}, \frac{1}{6}\right) .
\end{aligned}
$$

Analogously, for the CC coupling matrices $V_{L, R}$ :

$$
\begin{aligned}
& V_{L}=L \mathcal{V} L^{\dagger}, V_{R}=R \mathcal{V} R^{\dagger} ; \mathcal{V}_{L ; R}=\left(\begin{array}{cccc}
0 & \mathcal{V}^{X^{u}} & \mathbf{0}_{1 \times 6} & 0 \\
\mathbf{0}_{6 \times 1} & \mathbf{0}_{6 \times 6} & \mathcal{V}_{L ; R}^{\mathcal{T}} & \mathbf{0}_{6 \times 1} \\
\mathbf{0}_{6 \times 1} & \mathbf{0}_{6 \times 6} & \mathbf{0}_{6 \times 6} & \mathcal{V}^{\mathcal{B} X^{\prime d}} \\
0 & \mathbf{0}_{1 \times 6} & \mathbf{0}_{1 \times 6} & 0
\end{array}\right), \\
& \mathcal{V}^{X^{u} \mathcal{T}}=\left(\mathcal{V}^{\mathcal{B} X^{\prime d}}\right)^{\dagger}=(0,0,1,0,0,0),
\end{aligned}
$$

while $\mathcal{V}_{L}^{\mathcal{T B}}$ is a $6 \times 6$ matrix whose elements are null but for its $(1,1),(2,6)$ and $(6,2)$ entries with value 1 , and $\mathcal{V}_{R}^{\mathcal{T} \mathcal{B}}$ is a $6 \times 6$ matrix with null elements but for its $(2,6)$ and $(6,2)$ entries with value 1. 
T parameter. The contribution of the fermionic sector to the $T$ parameter, $\Delta T^{f}$, is given by [42]

$$
\begin{aligned}
\Delta T^{f}= & \frac{3}{16 \pi s_{W}^{2} c_{W}^{2}}\left\{\sum_{i j}\left[\left(\left(V_{L}^{i j}\right)^{2}+\left(V_{R}^{i j}\right)^{2}\right) \theta_{+}\left(\eta_{i}, \eta_{j}\right)+2 V_{L}^{i j} V_{R}^{i j} \theta_{-}\left(\eta_{i}, \eta_{j}\right)\right]\right. \\
& \left.-\frac{1}{2} \sum_{i j}\left[\left(\left(C_{L}^{i j}\right)^{2}+\left(C_{R}^{i j}\right)^{2}\right) \theta_{+}\left(\eta_{i}, \eta_{j}\right)+2 C_{L}^{i j} C_{R}^{i j} \theta_{-}\left(\eta_{i}, \eta_{j}\right)\right]\right\} \\
& -\frac{3}{16 \pi s_{W}^{2} c_{W}^{2} M_{Z}^{2}}\left(m_{t}^{2}+m_{b}^{2}-2 \frac{m_{t}^{2} m_{b}^{2}}{m_{t}^{2}-m_{b}^{2}} \ln \frac{m_{t}^{2}}{m_{b}^{2}}\right),
\end{aligned}
$$

where $m_{i}$ denotes the fermion masses, $m_{i} \equiv \mathcal{M}_{i i}^{\text {diag }}$, and $\eta_{i} \equiv m_{i}^{2} / M_{Z}^{2}$. The last line in this equation corresponds to the substraction of the SM contribution from the light fermions (top and bottom). The $\theta_{ \pm}$functions are defined as [42]:

$$
\begin{aligned}
& \theta_{+}\left(\eta_{1}, \eta_{2}\right)=\eta_{1}+\eta_{2}-\frac{2 \eta_{1} \eta_{2}}{\eta_{1}-\eta_{2}} \ln \frac{\eta_{1}}{\eta_{2}}-2\left(\eta_{1} \ln \eta_{1}+\eta_{2} \ln \eta_{2}\right)+\operatorname{div} \frac{\eta_{1}+\eta_{2}}{2} \\
& \theta_{-}\left(\eta_{1}, \eta_{2}\right)=2 \sqrt{\eta_{1} \eta_{2}}\left(\frac{\eta_{1}+\eta_{2}}{\eta_{1}-\eta_{2}} \ln \frac{\eta_{1}}{\eta_{2}}-2+\ln \left(\eta_{1} \eta_{2}\right)-\frac{\operatorname{div}}{2}\right) .
\end{aligned}
$$

S parameter. The fermionic contribution to $S, \Delta S^{f}$, can be computed following ref. [42],

$$
\begin{aligned}
\Delta S^{f}= & -\frac{1}{\pi} \sum_{i j}\left\{( C _ { L } ^ { i j } Y _ { L } ^ { i j } + C _ { R } ^ { i j } Y _ { R } ^ { i j } ) \left[-\frac{\operatorname{div}}{12}-\frac{5}{9}+\frac{\eta_{i}+\eta_{j}}{3}+\frac{\ln \left(\eta_{i} \eta_{j}\right)}{6}\right.\right. \\
& \left.+\frac{\eta_{i}-1}{12} f\left(\eta_{i}, \eta_{i}\right)+\frac{\eta_{j}-1}{12} f\left(\eta_{j}, \eta_{j}\right)-\frac{\chi_{+}\left(\eta_{i}, \eta_{j}\right)}{2}\right] \\
& \left.-\left(C_{L}^{i j} Y_{R}^{i j}+C_{R}^{i j} Y_{L}^{i j}\right)\left[2 \sqrt{\eta_{i} \eta_{j}}+\sqrt{\eta_{i} \eta_{j}} \frac{f\left(\eta_{i}, \eta_{i}\right)+f\left(\eta_{j}, \eta_{j}\right)}{4}+\frac{\chi_{-}\left(\eta_{i}, \eta_{j}\right)}{2}\right]\right\}-\Delta S_{\mathrm{SM}}^{f},
\end{aligned}
$$

with the functions $f\left(\eta_{1}, \eta_{2}\right)$ and $\chi_{ \pm}\left(\eta_{i}, \eta_{j}\right)$ as defined in ref. [42], "div" standing for the divergent contributions typically appearing in dimensional regularisation, and the last term corresponding to the substraction of the SM light (top and bottom) fermionic contributions. ${ }^{8}$

Anomalous $\boldsymbol{Z} \boldsymbol{b} \boldsymbol{b}$ coupling. We follow ref. [34] for the computation of the corrections to the $g_{L}^{b}$ parameter defined in eq. (4.4), $\delta g_{L}^{b}$. Only the top and bottom sectors will be taken into account as the mass generation mechanism for the lighter fermions are expected to have a lesser impact on EW precision tests since either the exotic fermions involved are much heavier or the Yukawa couplings connecting them to the SM fermions are much smaller. Moreover, the bottom quark mass will be neglected $\left(y_{1}^{\prime}=y_{2}^{\prime}=0\right) .{ }^{9}$ The fermion-gauge

\footnotetext{
${ }^{8}$ The SM fermionic contributions to $S$ and $T$ with only one generation of quarks follow from eq. (4.15) considering a two-component fermion field $\Psi^{\mathrm{SM}}=(t, b)$, with $\mathcal{M}^{\mathrm{SM}}=\operatorname{diag}\left(m_{t}, m_{b}\right)$ and coupling matrices $\mathcal{Q}^{\mathrm{SM}}=Y_{R}^{\mathrm{SM}}=\operatorname{diag}(+2 / 3,-1 / 3), C_{L}^{\mathrm{SM}}=\operatorname{diag}(+1,-1), Y_{L}^{\mathrm{SM}}=+\frac{1}{6} \mathbb{1}_{2 \times 2}, V_{L}^{\mathrm{SM}}=\operatorname{antidiag}(0,1), C_{R}^{\mathrm{SM}}=$ $V_{R}^{\mathrm{SM}}=\mathbb{O}_{2 \times 2}$.

${ }^{9}$ The cancellation of divergences in the computation of $\delta g_{L}^{b}$ has been verified in this approximation.
} 
couplings relevant to this case are the $\mathrm{Z}$ couplings for the charge $2 / 3$ and $-1 / 3$ sectors which can be read from eqs. (4.17) and (4.19), and the couplings to the $W^{ \pm}$boson between the $(2 / 3, R)$ and the $(-1 / 3, L)$ sectors (see the matrix $\mathcal{V}_{L}^{\mathcal{T} \mathcal{B}}$ defined after eq. (4.23)). In addition to the $\mathrm{NC}$ and $\mathrm{CC}$ couplings in eq. (4.15), the interactions of the charged longitudinal gauge boson components " $\pi_{i}$ " are needed,

$$
\mathcal{L}_{\pi^{ \pm}}=\bar{\Psi}^{\text {phys }} \frac{g}{\sqrt{2}}\left(W_{L} P_{L}+W_{R} P_{R}\right) \Psi^{\text {phys }} \pi^{+}+\text {h.c. }
$$

where

$$
W_{L}=R \mathcal{W}_{L} L^{\dagger}, \quad W_{R}=L \mathcal{W}_{R} R^{\dagger},
$$

with $\mathcal{W}_{L}$ and $\mathcal{W}_{R}$ being the mixing matrices in the interaction basis, given in the present model by

$$
\begin{aligned}
\mathcal{W}_{L ; R} & =\left(\begin{array}{cccc}
0 & \mathcal{W}_{L ; R}^{X^{u} \mathcal{T}} & \mathbf{0}_{1 \times 6} & 0 \\
\mathbf{0}_{6 \times 1} & \mathbf{0}_{6 \times 6} & \mathcal{W}_{L ; R}^{\mathcal{T}} & \mathbf{0}_{6 \times 1} \\
\mathbf{0}_{6 \times 1} & \mathbf{0}_{6 \times 6} & \mathbf{0}_{6 \times 6} & \mathcal{W}_{L ; R}^{\mathcal{B} X^{\prime d}} \\
0 & \mathbf{0}_{1 \times 6} & \mathbf{0}_{1 \times 6} & 0
\end{array}\right), \\
\mathcal{W}_{L ; R}^{X^{u} \mathcal{T}} & =\frac{\sqrt{2}}{g}\left(0,0,0,0,-y_{2} ;-y_{1}, 0\right),\left(\mathcal{W}_{L ; R}^{\mathcal{B} X^{\prime d}}\right)^{\dagger}=\frac{\sqrt{2}}{g}\left(0,0,0,0, y_{1}^{\prime} ; y_{2}^{\prime}, 0\right) .
\end{aligned}
$$

The $6 \times 6$ matrix $\mathcal{W}_{L}^{\mathcal{T} \mathcal{B}}$ in this equation has all elements null but for its $(5,6)$ and $(6,5)$ entries which take values $y_{1}$ and $-y_{2}^{\prime}$, respectively, while $\mathcal{W}_{R}^{\mathcal{T} \mathcal{B}}$ is a $6 \times 6$ matrix of null elements but for its $(5,6)$ and $(6,5)$ entries which take values $y_{2}$ and $-y_{1}^{\prime}$, respectively. In practice, only the entries connecting - after rotation - the charge $2 / 3$ fermions to $b_{L}$ enter the computation.

In the numerical analysis, the two sets of values considered earlier on for the numerical analysis of the pure scalar contributions will be retained: $\left(m_{\sigma}=2 \mathrm{TeV}, \sin _{\gamma}^{2}=0.04\right)$ and $\left(m_{\sigma}=6 \mathrm{TeV}, \sin _{\gamma}^{2}=0.06\right)$, both corresponding to $f=1 \mathrm{TeV}$ and within the soft breaking regime $\alpha, \beta<\lambda$, with the latter being kept within its perturbative range. ${ }^{10}$ Note that, for a $\sigma$ particle much heavier than the Higgs, values of $f$ below $700 \mathrm{GeV}$ would be difficult to accommodate experimentally as $\sin \gamma^{2} \simeq v^{2} / f^{2}$, see eq. (4.1). The exotic fermionic masses will be allowed to vary randomly between $800 \mathrm{GeV}$ and $\mathcal{O}(10 \mathrm{TeV})$, as the heavy top partners with electric charges $+5 / 3$ and $+2 / 3$ are bounded to be above $800-$ $1000 \mathrm{GeV}[12,13]$, depending on the dominant decay mode. In the light fermion sector, the top and bottom masses will be allowed to vary within the intervals $m_{t}=173 \pm 5 \mathrm{GeV}$ and $m_{b}=4.6 \pm 2 \mathrm{GeV}$, respectively, for illustrative purposes.

Figures 4 to 6 depict the points that satisfy a $\chi^{2}$ global fit to the precision pseudoobservables $S, T$ and $\delta g_{L}^{b}$, where the blue, green, and red points are the allowed $1 \sigma, 2 \sigma$ and $3 \sigma$ regions, respectively, while gray points lie above the $3 \sigma$ limit. The central values, uncertainties and correlation matrix are taken from ref. [35]. The ellipses drawn in the $\Delta S-\Delta T$ plane in figures 4 and 5 are the projection for $\Delta g_{b}^{L}=0$, while those in the

\footnotetext{
${ }^{10} m_{\sigma}=4 \pi f$ is roughly where perturbativity is lost in chiral perturbation theory [10].
} 


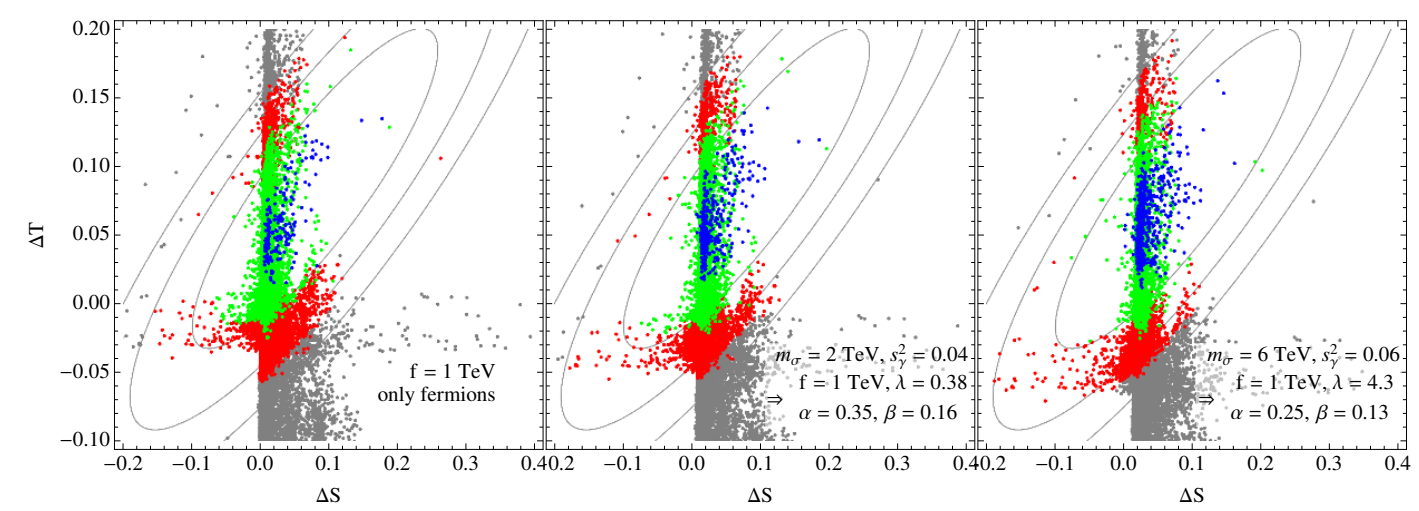

Figure 5. Combined contributions to $S$ and $T$ from the scalar sector and the exotic fermionic sector. The blue, green and red points are allowed at $1,2,3 \sigma$ by the combined $\left(S, T, g_{L}^{b}\right)$ fit, while gray points are outside the $3 \sigma$ region.

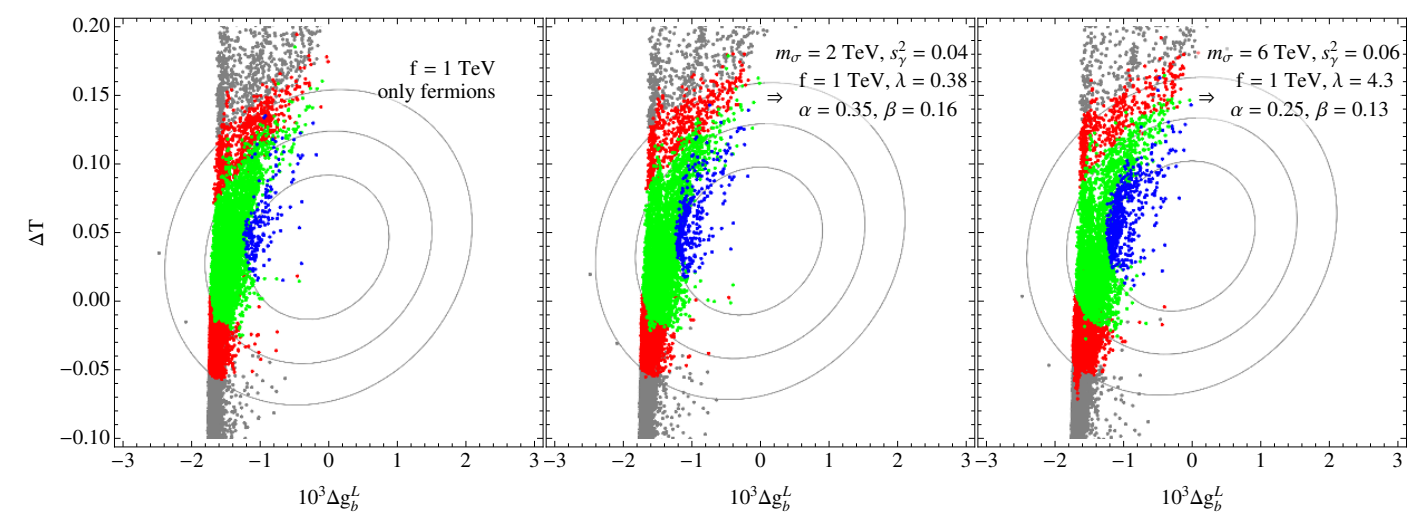

Figure 6. Scalar and fermionic impact on the $T$ parameter and on the $Z-b_{L}-b_{L}$ coupling $g_{b}^{L}$.

$\Delta T-\Delta g_{b}^{L}$ plane in figure 6 use the $\Delta S$ value coming from the scalar sector. The latter is a good approximation since $S$ gets in practice a very small correction from the heavy fermions, as seen in figure 5.

$\mathbf{S}$ versus T. The fermion sector can lead to large deviations in the value of the $T$ parameter. In figure 4 and in the first panel of figure 5 only the fermionic contributions are depicted. The last two panels in figure 5 show the fermion plus scalar combined results: the lighter the $\sigma$ particle, the less tension follows with respect to electroweak precision data, in particular due to the impact on $\Delta T$, although it is to be noted that even for large $m_{\sigma}$ fermionic contributions can bring $(S, T)$ within the experimentally allowed region.

The sign of the fermionic contributions to $S$ and $T$ can be largely understood in terms of the light-heavy fermion mixings and the mass hierarchy between the heavy eigenstates. For instance, large mixing values with a heavy singlet are known to induce large positive contributions to $\Delta T$, as pointed out in ref. [34], as a result from the custodial symmetry 

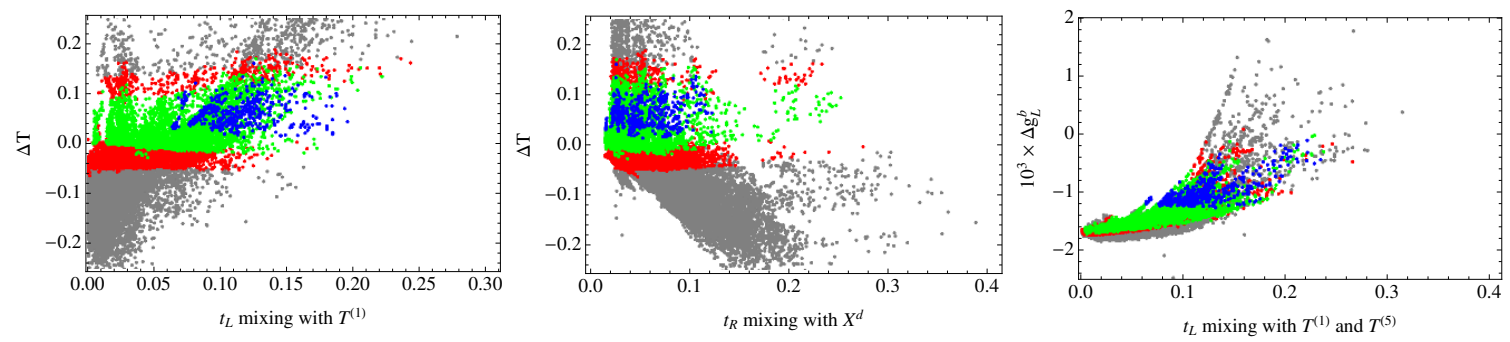

Figure 7. Examples of correlations between the fermion mixing strength and electroweak precision measurements. The label " $t_{L, R}$ mixing with $\Psi_{1}, \Psi_{2}, \ldots$ " indicates $\left(\left|U_{1}^{L, R}\right|^{2}+\left|U_{2}^{L, R}\right|^{2}+\ldots\right)^{1 / 2}$, where $U^{L, R}$ indicates the left or right rotation that diagonalizes the mass matrix.

being broken by the singlet-doublet mixing. It is possible to illustrate the analysis more in detail following ref. [43], which uses a different fermionic but nonetheless illuminating embedding. They consider heavy vector fermions which couple directly to both the light doublet $q$ and the light singlet $q_{R}$. When only a heavy singlet is present, the expected contributions to $\Delta T$ and $\Delta S$ are both significant (though the first are more important) and positive for the regime we consider, see their eq. (32). Instead, when only a heavy vector doublet was taken into account, the sign of the correction to the oblique parameters was proportional to the sign of the mass splitting between the heavy eigenstates with charge $2 / 3$ and $-1 / 3$, resulting in sizeable contributions to $\Delta T$ and very small to $\Delta S$.

It is not possible to apply those conclusions in ref. [43] directly here, though, as in our setup the light fermion mass generation involves necessarily and simultaneously both a heavy doublet and a heavy singlet, see eq. (3.6): the light doublet $q$ mixes directly only with the heavy doublet $Q$, while $q_{R}$ mixes with $T_{1}$. Nevertheless, the mainly positive fermionic corrections to $\Delta S$ found are consistent with being dominated by the participation of a heavy singlet. The results, in figure 7 show indeed that a large mixing between $t_{L}$ and the singlet $T^{(1)}$ leads to a positive $\Delta T$ (left panel) while the negative corrections to $\Delta T$ obtained are consistent instead with a large mixing between $t_{R}$ and the doublet component $X^{d}$ (middle panel).

$\mathbf{T}$ versus $\boldsymbol{g}_{L}^{b}$. The deviations induced in the $Z b b$ coupling provide additional bounds: even if the model parameters do not impact on $\Delta g_{b}^{L}$ at tree level, the top partners may induce at loop level deviations from the SM value. Figure 6 depicts the purely fermionic and the scalar plus fermion combined contributions in the $T-g_{L}^{b}$ parameter space. Finally, the right panel of figure 7 shows a sizeable and positive impact on $g_{L}^{b}$ of the mixing between $t_{L}$ and the charge $2 / 3$ heavy singlets $T^{(1)}$ and $T^{(5)}$.

As a final remark, there are considerable mixings in the fermion sector for which the dominant effects go schematically as $\tan \theta_{i j} \sim \Lambda_{i} / M_{j}$. It could be thus suspected that large deviations in the $W t b$ coupling should occur. However, these rotations are mainly driven by the $\mathrm{SO}(5)$ breaking couplings $\Lambda_{i}$ and $\Lambda_{i}^{\prime}$, which are custodial symmetry preserving. Therefore, a large rotation in the top sector is mostly compensated by a corresponding one in the bottom sector, leading to practically no deviation in $V_{t b}$. 


\subsection{Higgs and $\sigma$ coupling to gluons}

This section and the next one deal with the scalar to photons and to gluons effective couplings, arising at one-loop level. Define the scalar-gluon-gluon amplitudes $h g g(\sigma g g)$ as

$$
\mathcal{A}_{h(\sigma)} \equiv \mathcal{A}_{h(\sigma) \leftrightarrow g g}\left(m_{h(\sigma)}^{2}\right)=-i \frac{\alpha_{s}}{\pi} g_{h(\sigma)}\left(p \cdot k g^{\mu \nu}-p^{\mu} k^{\nu}\right) \delta^{a b},
$$

where $g_{h}$ and $g_{\sigma}$ are scale dependent functions that parametrize the amplitude strength, $\alpha_{s}=g_{s}^{2} / 4 \pi$ with $g_{s}$ denoting the QCD coupling constant, $p$ and $k$ stand for the gluon fourmomenta, and $a, b$ are color indices. In the case of the SM, the $h g g$ coupling is induced only at one loop level and the amplitude is dominated by the top quark,

$$
g_{h}^{\mathrm{SM}}=\left(\frac{y_{t}}{\sqrt{2}}\right) \frac{1}{m_{t}} I\left(\frac{m_{h}^{2}}{m_{t}^{2}}\right),
$$

where $y_{t}$ is the top Yukawa coupling $\left(m_{t} \equiv y_{t} v / \sqrt{2}\right)$ and $I\left(m_{h}^{2} / m_{t}^{2}\right) / m_{t}$ is the loop factor with

$$
I\left(\frac{q^{2}}{m^{2}}\right)=\int_{0}^{1} d x \int_{0}^{1-x} d z \frac{1-4 x z}{1-x z \frac{q^{2}}{m^{2}}} \approx\left\{\begin{array}{lll}
1 / 3 & \text { for } & m^{2} \gg q^{2} \\
0 & \text { for } & m^{2} \ll q^{2}
\end{array}\right\} .
$$

The SM bottom contribution corresponds to $I\left(m_{h}^{2} / m_{b}^{2}\right) \approx 10^{-2}$ and is thus usually neglected. ${ }^{11}$

There are no direct $h g g$ or $\sigma g g$ couplings in the Lagrangian discussed here, but effective $h g g$ and $\sigma g g$ interactions arise via fermion loops. Expanding the global field-dependent mass matrix $\mathcal{M}(h, \sigma)$ in eqs. (3.9)-(3.12) around the scalar field vevs, $v$ and $v_{\sigma}$, and defining the following constant matrices

$$
\overline{\mathcal{M}} \equiv \mathcal{M}\left(v, v_{\sigma}\right) \quad,\left.\quad \frac{\partial \overline{\mathcal{M}}}{\partial h} \equiv \frac{\partial \mathcal{M}(h, \sigma)}{\partial h}\right|_{\substack{h=v \\ \sigma=v_{\sigma}}} \quad,\left.\quad \frac{\partial \overline{\mathcal{M}}}{\partial \sigma} \equiv \frac{\partial \mathcal{M}(h, \sigma)}{\partial \sigma}\right|_{\substack{h=v \\ \sigma=v_{\sigma}}} \quad,
$$

the fermionic mass Lagrangian eq. (3.9) can be written as

$$
-\mathcal{L}_{Y}=\bar{\Psi}_{L} \overline{\mathcal{M}} \Psi_{R}+\hat{h} \bar{\Psi}_{L} \frac{\partial \overline{\mathcal{M}}}{\partial h} \Psi_{R}+\hat{\sigma} \bar{\Psi}_{L} \frac{\partial \overline{\mathcal{M}}}{\partial \sigma} \Psi_{R}+\text { h.c. }
$$

where $\hat{h}$ and $\hat{\sigma}$ are the unrotated scalar fluctuations, see eq. (2.14). Performing the rotation to the fermionic mass eigenstate basis $\left\{\Psi_{i} \rightarrow \Psi_{i}^{\text {phys }}\right\}$,

$$
(\overline{\mathcal{M}})_{i j} \rightarrow m_{i} \delta_{i j} \quad, \quad\left(\frac{\partial \overline{\mathcal{M}}}{\partial h}\right)_{i j} \rightarrow\left(Y_{h}\right)_{i j} \quad, \quad\left(\frac{\partial \overline{\mathcal{M}}}{\partial \sigma}\right)_{i j} \rightarrow\left(Y_{\sigma}\right)_{i j}
$$

where $m_{i}, Y_{h}$ and $Y_{\sigma}$ are respectively the masses and the couplings to the unrotated scalars fields $\hat{h}, \hat{\sigma}$ of the physical fermionic states. ${ }^{12}$ For simplicity, CP invariance will be assumed in what follows.

\footnotetext{
${ }^{11}$ The large mass limit in the integral is customarily applied for $m_{h}<2 m_{i}$, which includes the top case.

${ }^{12}$ For instance, in this notation $\left(Y_{h}\right)_{t t}=y_{t} / \sqrt{2}$.
} 
It is straightforward to obtain the physical $h \leftrightarrow g g$ and $\sigma \leftrightarrow g g$ amplitudes combining those involving the unrotated $\hat{h}$ and $\hat{\sigma}$ fields. The latter will require the substitution of the SM loop factor in eq. (4.3) as follows,

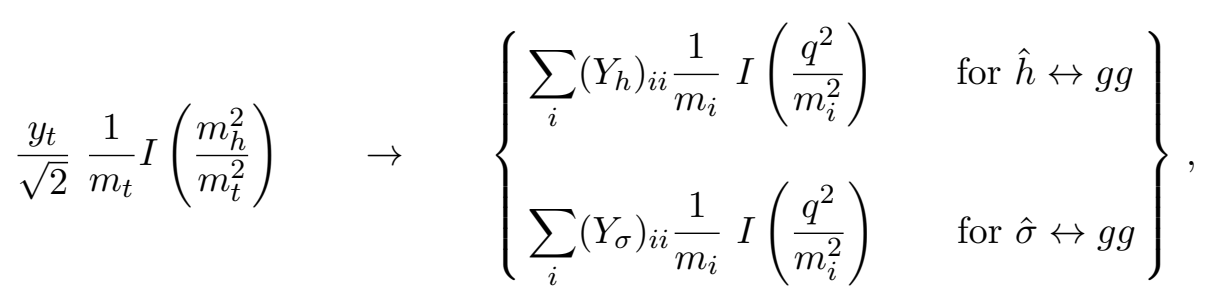

where $q^{2}=m_{h}^{2}$ for $h \leftrightarrow g g$ on-shell transitions, while $q^{2}=m_{\sigma}^{2}$ for $\sigma \leftrightarrow g g$ on-shell transitions, and where the sum runs over all colored fermion species present in the model.

$\boldsymbol{h} \leftrightarrow \boldsymbol{g g}$ transitions. If all fermion masses were much larger than $m_{h}$, it would be possible to simply factorize the constant integral outside the sum as follows:

$$
\sum_{i} \frac{\left(Y_{h}\right)_{i i}}{m_{i}} I\left(\frac{m_{h}^{2}}{m_{i}^{2}}\right) \approx \frac{1}{3} \sum_{i} \frac{\left(Y_{h}\right)_{i i}}{m_{i}}=\frac{1}{6} \frac{d}{d h} \log \operatorname{det}\left(\overline{\mathcal{M}} \overline{\mathcal{M}}^{\dagger}\right),
$$

where the last term is written in the original unrotated fermionic basis since trace and determinant are invariant under a change of basis. All fermions in the model under consideration are indeed much heavier than the Higgs particle but for the bottom, whose loop contribution $I\left(m_{h}^{2} / m_{b}^{2}\right)$ is negligible. Therefore the false "heavy" bottom contribution included in eq. (4.36) should be removed at energies $q^{2} \approx m_{h}^{2}$, resulting in the following effective couplings at the scale $m_{h}$ :

$$
\begin{aligned}
& \left.g_{\hat{h}}\left(m_{h}^{2}\right) \approx \frac{1}{6} \frac{d}{d h}\left(\log \operatorname{det}\left(\mathcal{M} \mathcal{M}^{\dagger}\right)-\log \left(m_{b}(h, \sigma) m_{b}^{*}(h, \sigma)\right)\right)\right|_{\substack{h=v \\
\sigma=v_{\sigma}}} \\
& =\frac{1}{3 v}+O\left(\frac{v}{M_{1}^{\prime} M_{5}^{\prime}}\right), \\
& \left.g_{\hat{\sigma}}\left(m_{h}^{2}\right) \approx \frac{1}{6} \frac{d}{d \sigma}\left(\log \operatorname{det}\left(\mathcal{M} \mathcal{M}^{\dagger}\right)-\log \left(m_{b}(h, \sigma) m_{b}^{*}(h, \sigma)\right)\right)\right|_{\substack{h=v \\
\sigma=v_{\sigma}}} \\
& =-\frac{y_{2}}{3 M_{5}} \frac{\Lambda_{2}}{\Lambda_{3}}+O\left(\frac{v_{\sigma}}{M_{1}^{\prime} M_{5}^{\prime}}, \frac{v_{\sigma}}{M_{5}^{2}}\right),
\end{aligned}
$$

where the eigenvalue of the field dependent mass matrix corresponding to the bottom quark reads:

$$
m_{b}(h, \sigma)=\frac{y_{1}^{\prime} \Lambda_{1}^{\prime} \Lambda_{3}^{\prime}-y_{1}^{\prime} y_{2}^{\prime} \Lambda_{1}^{\prime} \Lambda_{2}^{\prime} \sigma / M_{5}^{\prime}}{M_{1}^{\prime} M_{5}^{\prime}-y_{1}^{\prime} y_{2}^{\prime}\left(h^{2}+\sigma^{2}\right)} \frac{h}{\sqrt{2}} .
$$

The $h \leftrightarrow g g$ amplitude is then given by eq. (4.31), with

$$
g_{h} \equiv g_{\hat{h}}\left(m_{h}^{2}\right) \cos \gamma-g_{\hat{\sigma}}\left(m_{h}^{2}\right) \sin \gamma .
$$

In the limit $m_{t} \gg m_{h}$, the $h g g$ effective coupling is exactly as in the SM. The contribution from the heavy vector-like quarks tends to cancel out for bare vector-like masses substantially larger than $v$, a result well-known in the literature. 
$\boldsymbol{\sigma} \leftrightarrow \boldsymbol{g g}$ transitions. With analogous procedure, the $\sigma g g$ amplitude can be obtained using eq. (4.35) for $q^{2}=m_{\sigma}^{2}$. The difference with the previous case is that now the top quark is lighter or comparable in mass to $\sigma$ and it cannot be integrated out, that is $m_{b} \ll$ $m_{t}, m_{\sigma} \ll m_{i}$, where here $m_{i}$ denotes the heavy fermion masses, and in consequence it is necessary to subtract the bottom contribution and to take into account the $q^{2}$ dependence in the top loop. In the approximation $I\left(m_{\sigma}^{2} / m_{b}^{2}\right) \approx 0$, it results

$$
\begin{aligned}
& \left.g_{\hat{h}}\left(m_{\sigma}^{2}\right) \approx \frac{1}{6} \frac{d}{d h}\left(\log \operatorname{det}\left(\mathcal{M} \mathcal{M}^{\dagger}\right)-\log \left(m_{t}(h, \sigma) m_{t}^{*}(h, \sigma)\right)-\log \left(m_{b}(h, \sigma) m_{b}^{*}(h, \sigma)\right)\right)\right|_{\substack{h=v \\
\sigma=v_{\sigma}}} \\
& +\frac{1}{v} I\left(\frac{m_{\sigma}^{2}}{m_{t}^{2}}\right) \\
& =\frac{1}{v} I\left(\frac{m_{\sigma}^{2}}{m_{t}^{2}}\right)-\frac{2}{3} v\left(\frac{y_{1} y_{2}}{M_{1} M_{5}}+\frac{y_{1}^{\prime} y_{2}^{\prime}}{M_{1}^{\prime} M_{5}^{\prime}}\right)+O\left(\frac{v v_{\sigma}^{2}}{M_{1}^{2} M_{5}^{2}}, \frac{v v_{\sigma}^{2}}{M_{1}^{\prime 2} M_{5}^{\prime 2}}\right) \text {, } \\
& \left.g_{\hat{\sigma}}\left(m_{\sigma}^{2}\right) \approx \frac{1}{6} \frac{d}{d \sigma}\left(\log \operatorname{det}\left(\mathcal{M} \mathcal{M}^{\dagger}\right)-\log \left(m_{t}(h, \sigma) m_{t}^{*}(h, \sigma)\right)-\log \left(m_{b}(h, \sigma) m_{b}^{*}(h, \sigma)\right)\right)\right|_{\substack{h=v \\
\sigma=v_{\sigma}}} \\
& -\frac{y_{2}}{M_{5}} \frac{\Lambda_{2}}{\Lambda_{3}} I\left(\frac{m_{\sigma}^{2}}{m_{t}^{2}}\right) \\
& =-\frac{y_{2}}{M_{5}} \frac{\Lambda_{2}}{\Lambda_{3}} I\left(\frac{m_{\sigma}^{2}}{m_{t}^{2}}\right)-\frac{2}{3} v_{\sigma}\left(\frac{y_{1} y_{2}}{M_{1} M_{5}}+\frac{y_{1}^{\prime} y_{2}^{\prime}}{M_{1}^{\prime} M_{5}^{\prime}}\right)+O\left(\frac{v_{\sigma}^{3}}{M_{1}^{2} M_{5}^{2}}, \frac{v_{\sigma}^{3}}{M_{1}^{\prime 2} M_{5}^{\prime 2}}\right),
\end{aligned}
$$

where the eigenvalue of the field dependent mass matrix corresponding to the top quark reads

$$
m_{t}(h, \sigma)=\frac{y_{1} \Lambda_{1} \Lambda_{3}-y_{1} y_{2} \Lambda_{1} \Lambda_{2} \sigma / M_{5}}{M_{1} M_{5}-y_{1} y_{2}\left(h^{2}+\sigma^{2}\right)} \frac{h}{\sqrt{2}} .
$$

Note that the dominant contribution to the $\sigma g g$ effective coupling requires both $y_{1}$ and $y_{2}$ to be non-vanishing. In contrast, the dominant contribution to the top quark mass is proportional to $y_{1}$ but independent of $y_{2}$.

The $\sigma \leftrightarrow g g$ amplitude is finally given by eqs. (4.31), (4.40) and (4.41), with

$$
g_{\sigma} \equiv g_{\hat{h}}\left(m_{\sigma}^{2}\right) \sin \gamma+g_{\hat{\sigma}}\left(m_{\sigma}^{2}\right) \cos \gamma .
$$

The matrix elements modulus square for $g g \rightarrow h$ and $g g \rightarrow \sigma$, averaged over the polarisations of the initial state, are then given by

$$
\begin{aligned}
& \overline{\left|\mathcal{A}_{h}\right|^{2}}=\frac{\alpha_{S}^{2} m_{h}^{4}}{64 \pi^{2}} g_{h}^{2}, \\
& \overline{\left|\mathcal{A}_{\sigma}\right|^{2}}=\frac{\alpha_{S}^{2} m_{\sigma}^{4}}{64 \pi^{2}} g_{\sigma}^{2} .
\end{aligned}
$$

In terms of those amplitudes, the cross section at the parton level can be expressed as

$$
\begin{aligned}
\sigma_{\text {part }}(g g \rightarrow h) & =\overline{\left|\mathcal{A}_{h}\right|^{2}} \frac{\pi}{s_{\text {part }}} \delta\left(s_{\text {part }}-m_{h}^{2}\right) \\
& =\overline{\left|\mathcal{A}_{h}\right|^{2}} \frac{\pi}{\tau s^{2}} \delta\left(\tau-\frac{m_{h}^{2}}{s}\right),
\end{aligned}
$$


where as usual $s_{\text {part }}$ denotes the center-of-mass energy at the parton level $s_{\text {part }}=\tau s$. A similar expression holds for $\sigma_{\text {part }}(g g \rightarrow \sigma)$. By convoluting the cross-section with the gluon densities $G(x)$ we finally obtain

$$
\begin{aligned}
& \sigma(p p \rightarrow h)=\overline{\left|\mathcal{A}_{h}\right|^{2}} \frac{\pi}{m_{h}^{2} s} \times \int_{m_{h}^{2} / s}^{1} \frac{d x}{x} G(x) G\left(\frac{m_{h}^{2}}{s x}\right), \\
& \sigma(p p \rightarrow \sigma)=\overline{\left|\mathcal{A}_{\sigma}\right|^{2}} \frac{\pi}{m_{\sigma}^{2} s} \times \int_{m_{\sigma}^{2} / s}^{1} \frac{d x}{x} G(x) G\left(\frac{m_{\sigma}^{2}}{s x}\right) .
\end{aligned}
$$

In resume, for very heavy fermion partners the $h$-gluon-gluon transitions are dominated by the top quark contribution, while they have a more significant impact on $\sigma$-gluon-gluon transitions.

\subsection{Higgs and $\sigma$ decay into $\gamma \gamma$}

There are no direct $h \gamma \gamma$ or $\sigma \gamma \gamma$ couplings in our Lagrangian. They arise instead as effective interactions from loops of fermions and, in the case of $h \gamma \gamma$, also of massive vector bosons. As in the case of $h$ and $\sigma$ production via $g g$ fusion, we distinguish between mass eigenstates $h, \sigma$ and the unrotated (interaction ) eigenstates $\hat{h}, \hat{\sigma}$.

Let the scalar-photon-photon amplitudes $h \gamma \gamma$ and $\sigma \gamma \gamma$ be defined as

$$
\mathcal{A}_{h(\sigma) \leftrightarrow \gamma \gamma}\left(m_{h(\sigma)}^{2}\right)=i \frac{\alpha}{\pi} \Omega_{h(\sigma)}\left(p \cdot k g^{\mu \nu}-p^{\mu} k^{\nu}\right) \delta^{a b},
$$

where again $\Omega_{h}$ and $\Omega_{\sigma}$ are scale dependent functions. The decay amplitudes are then given by

$$
\Gamma(h \rightarrow \gamma \gamma)=\frac{\alpha^{2} m_{h}^{3}}{64 \pi^{3}}\left|\Omega_{h}\right|^{2}, \quad \Gamma(\sigma \rightarrow \gamma \gamma)=\frac{\alpha^{2} m_{\sigma}^{3}}{64 \pi^{3}}\left|\Omega_{\sigma}\right|^{2} .
$$

In the model under study, the contributions can again be decomposed as

$$
\begin{aligned}
& \Omega_{h}=\cos \gamma \Omega_{\hat{h}}\left(m_{h}^{2}\right)-\sin \gamma \Omega_{\hat{\sigma}}\left(m_{h}^{2}\right), \\
& \Omega_{\sigma}=\sin \gamma \Omega_{\hat{h}}\left(m_{\sigma}^{2}\right)+\cos \gamma \Omega_{\hat{\sigma}}\left(m_{\sigma}^{2}\right) .
\end{aligned}
$$

While both unrotated scalar fields $\hat{h}$ and $\hat{\sigma}$ couple to fermions, only $\hat{h}$ couples to the $W$ boson,

$$
\Omega_{\hat{h}}\left(q^{2}\right)=\Omega_{\hat{h}}^{F}\left(q^{2}\right)+\Omega_{\hat{h}}^{W}\left(q^{2}\right) \quad, \quad \Omega_{\hat{\sigma}}\left(q^{2}\right)=\Omega_{\hat{\sigma}}^{F}\left(q^{2}\right),
$$

where the superscripts $F$ and $W$ stand for fermionic and gauge contributions, respectively. The latter is akin to the SM one, that is,

$$
\Omega_{\hat{h}}^{W}\left(q^{2}\right)=\frac{g^{2} v}{8 m_{W}^{2}} I_{W}\left(\frac{4 m_{W}^{2}}{q^{2}}\right),
$$

where the factor $g^{2} v$ results from the Higgs $-W W$ vertex, and the remaining part $I_{W} / 8 m_{W}^{2}$ results from the kinematics of the loop integral

$$
I_{W}(x)=2+3 x+3 x(2-x) f(x), \quad f(x)=\left\{\begin{array}{ll}
\arcsin ^{2}(1 / \sqrt{x}) & x \geq 1 \\
-\frac{1}{4}\left[\log \frac{1+\sqrt{1-x}}{1-\sqrt{1-x}}-i \pi\right]^{2} & x<1
\end{array} .\right.
$$


$\boldsymbol{h} \leftrightarrow \boldsymbol{\gamma} \gamma$ transitions. At the Higgs mass scale, the SM $\hat{h} W W$ coupling in eq. (4.48) is given by

$$
\Omega_{\hat{h}}^{W}\left(m_{h}^{2}\right) \approx \frac{4.2}{v} .
$$

The SM quark contributions are in turn given by

$$
\Omega_{h}^{\mathrm{SM}, F}=-6 \sum_{f} Q_{f}^{2}\left(\frac{y_{f}}{\sqrt{2}}\right) \frac{1}{m_{f}} I\left(\frac{m_{h}^{2}}{m_{f}^{2}}\right) \approx-\frac{8}{9} \frac{1}{v},
$$

where $y_{f}$ is the fermion Yukawa coupling, $m_{f}=y_{f} v / \sqrt{2}$, and the remaining factor $I / m_{f}$ results from the loop integral. The last expression in eq. (4.51) corresponds to the top contribution, which dominates the SM fermionic contribution. The SM decay $h \rightarrow \gamma \gamma$ decay rate is as given in eq. (4.46) with $\Omega_{h}=\Omega_{h}^{\mathrm{SM}, W}+\Omega_{h}^{\mathrm{SM}, F}$. In the model under consideration these expressions for the quark contributions to the $h \rightarrow \gamma \gamma$ transitions are generalized as follows, in analogy with the $g g$ fusion analysis above,

$$
\begin{aligned}
& \Omega_{\hat{h}}^{F}\left(m_{h}^{2}\right)=-2 \sum_{f} N_{C}^{f} Q_{f}^{2} \omega_{f}^{h}\left(m_{h}^{2}\right)=-2\left[3\left(\frac{2}{3}\right)^{2} \omega_{2 / 3}^{h}\left(m_{h}^{2}\right)+3\left(-\frac{1}{3}\right)^{2} \omega_{-1 / 3}^{h}\left(m_{h}^{2}\right)\right], \\
& \Omega_{\hat{\sigma}}^{F}\left(m_{h}^{2}\right)=-2 \sum_{f} N_{C}^{f} Q_{f}^{2} \omega_{f}^{\sigma}\left(m_{h}^{2}\right)=-2\left[3\left(\frac{2}{3}\right)^{2} \omega_{2 / 3}^{\sigma}\left(m_{h}^{2}\right)+3\left(-\frac{1}{3}\right)^{2} \omega_{-1 / 3}^{\sigma}\left(m_{h}^{2}\right)\right],
\end{aligned}
$$

where $N_{C}^{f}$ is the number of colors of a given quark species $f$, and $\omega_{f}^{h}$ are scale-dependent functions, which for charge $2 / 3$ and $-1 / 3$ fermions read

$$
\begin{aligned}
& \left.\omega_{2 / 3}^{h}\left(m_{h}^{2}\right) \equiv \frac{1}{6} \frac{d}{d h}\left(\log \operatorname{det}\left(\mathcal{M}_{\mathcal{T}} \mathcal{M}_{\mathcal{T}}^{\dagger}\right)\right)\right|_{\substack{h=v \\
\sigma=v_{\sigma}}}=\frac{1}{3 v}, \\
& \left.\omega_{-1 / 3}^{h}\left(m_{h}^{2}\right) \equiv \frac{1}{6} \frac{d}{d h}\left(\log \operatorname{det}\left(\mathcal{M}_{\mathcal{B}} \mathcal{M}_{\mathcal{B}}^{\dagger}\right)-\log \left(m_{b}(h, \sigma) m_{b}^{*}(h, \sigma)\right)\right)\right|_{\substack{h=v \\
\sigma=v_{\sigma}}} \\
& =-\frac{2}{3} v \frac{y_{1}^{\prime} y_{2}^{\prime}}{M_{1}^{\prime} M_{5}^{\prime}}+\mathcal{O}\left(\frac{v v_{\sigma}^{2}}{M_{1}^{\prime 2} M_{5}^{\prime 2}}\right) .
\end{aligned}
$$

For the $\omega^{\sigma}$ functions, it holds instead

$$
\begin{aligned}
& \left.\omega_{2 / 3}^{\sigma}\left(m_{h}^{2}\right) \equiv \frac{1}{6} \frac{d}{d \sigma}\left(\log \operatorname{det}\left(\mathcal{M}_{\mathcal{T}} \mathcal{M}_{\mathcal{T}}^{\dagger}\right)\right)\right|_{\substack{h=v \\
\sigma=v_{\sigma}}}=-\frac{y_{2}}{3 M_{5}} \frac{\Lambda_{2}}{\Lambda_{3}}+\mathcal{O}\left(\frac{v_{\sigma}}{M_{5}^{2}}\right) \\
& \left.\omega_{-1 / 3}^{\sigma}\left(m_{h}^{2}\right) \equiv \frac{1}{6} \frac{d}{d \sigma}\left(\log \operatorname{det}\left(\mathcal{M}_{\mathcal{B}} \mathcal{M}_{\mathcal{B}}^{\dagger}\right)-\log \left(m_{b}(h, \sigma) m_{b}^{*}(h, \sigma)\right)\right)\right|_{\substack{h=v \\
\sigma=v_{\sigma}}} \\
& =-\frac{2}{3} \frac{v_{\sigma} y_{1}^{\prime} y_{2}^{\prime}}{M_{1}^{\prime} M_{5}^{\prime}}+\mathcal{O}\left(\frac{v_{\sigma}^{3}}{M_{1}^{\prime 2} M_{5}^{\prime 2}}\right) \text {. }
\end{aligned}
$$

In these expressions the bottom contribution was neglected, while it has been assumed $m_{h} \ll m_{i}$ for the top mass and all other exotic fermion masses $m_{i}$. 
$\sigma \leftrightarrow \gamma \gamma$ transitions. Similarly, for $\sigma$ decaying into two photons the contributions for the unrotated field $\hat{\sigma}$ are given by

$$
\begin{aligned}
& \Omega_{\hat{\sigma}}^{F}\left(m_{\sigma}^{2}\right)=-2 \sum_{f} N_{C}^{f} Q_{f}^{2} \omega_{f}^{\sigma}\left(m_{\sigma}^{2}\right)=-2\left[3\left(\frac{2}{3}\right)^{2} \omega_{2 / 3}^{\sigma}\left(m_{\sigma}^{2}\right)+3\left(-\frac{1}{3}\right)^{2} \omega_{-1 / 3}^{\sigma}\left(m_{\sigma}^{2}\right)\right] \\
& \Omega_{\hat{h}}^{F}\left(m_{\sigma}^{2}\right)=-2 \sum_{f} N_{C}^{f} Q_{f}^{2} \omega_{f}^{h}\left(m_{\sigma}^{2}\right)=-2\left[3\left(\frac{2}{3}\right)^{2} \omega_{2 / 3}^{h}\left(m_{\sigma}^{2}\right)+3\left(-\frac{1}{3}\right)^{2} \omega_{-1 / 3}^{h}\left(m_{\sigma}^{2}\right)\right]
\end{aligned}
$$

where

$$
\begin{aligned}
\omega_{2 / 3}^{\sigma}\left(m_{\sigma}^{2}\right) & \left.\equiv \frac{1}{6} \frac{d}{d \sigma}\left(\log \operatorname{det}\left(\mathcal{M}_{\mathcal{T}} \mathcal{M}_{\mathcal{T}}^{\dagger}\right)-\log \left(m_{t}(h, \sigma) m_{t}^{*}(h, \sigma)\right)\right)\right|_{\substack{h=v \\
\sigma=v_{\sigma}}}-\frac{y_{2}}{M_{5}} \frac{\Lambda_{2}}{\Lambda_{3}} I\left(\frac{m_{\sigma}^{2}}{m_{t}^{2}}\right) \\
& =-\frac{y_{2}}{M_{5}} \frac{\Lambda_{2}}{\Lambda_{3}} I\left(\frac{m_{\sigma}^{2}}{m_{t}^{2}}\right)-\frac{2}{3} v_{\sigma} \frac{y_{1} y_{2}}{M_{1} M_{5}}+\mathcal{O}\left(\frac{v_{\sigma}^{3}}{M_{1}^{2} M_{5}^{2}}\right), \\
\omega_{-1 / 3}^{\sigma}\left(m_{\sigma}^{2}\right) & \left.\equiv \frac{1}{6} \frac{d}{d \sigma}\left(\log \operatorname{det}\left(\mathcal{M}_{\mathcal{B}} \mathcal{M}_{\mathcal{B}}^{\dagger}\right)-\log \left(m_{b}(h, \sigma) m_{b}^{*}(h, \sigma)\right)\right)\right|_{\substack{h=v \\
\sigma=v_{\sigma}}} \\
& =-\frac{2}{3} v_{\sigma} \frac{y_{1}^{\prime} y_{2}^{\prime}}{M_{1}^{\prime} M_{5}^{\prime}}+\mathcal{O}\left(\frac{v_{\sigma}^{3}}{M_{1}^{\prime 2} M_{5}^{\prime 2}}\right),
\end{aligned}
$$

while for $\omega^{h}\left(m_{\sigma}^{2}\right)$ it results

$$
\begin{aligned}
& \left.\omega_{2 / 3}^{h}\left(m_{\sigma}^{2}\right) \equiv \frac{1}{6} \frac{d}{d h}\left(\log \operatorname{det}\left(\mathcal{M}_{\mathcal{T}} \mathcal{M}_{\mathcal{T}}^{\dagger}\right)-\log \left(m_{t}(h, \sigma) m_{t}^{*}(h, \sigma)\right)\right)\right|_{\substack{h=v \\
\sigma=v_{\sigma}}}+\frac{1}{v} I\left(\frac{m_{\sigma}^{2}}{m_{t}^{2}}\right) \\
& =\frac{1}{v} I\left(\frac{m_{\sigma}^{2}}{m_{t}^{2}}\right)-\frac{2}{3} v \frac{y_{1} y_{2}}{M_{1} M_{5}}+\mathcal{O}\left(\frac{v v_{\sigma}^{2}}{M_{1}^{2} M_{5}^{2}}\right) \\
& \left.\omega_{-1 / 3}^{h}\left(m_{\sigma}^{2}\right) \equiv \frac{1}{6} \frac{d}{d h}\left(\log \operatorname{det}\left(\mathcal{M}_{\mathcal{B}} \mathcal{M}_{\mathcal{B}}^{\dagger}\right)-\log \left(m_{b}(h, \sigma) m_{b}^{*}(h, \sigma)\right)\right)\right|_{\substack{h=v \\
\sigma=v_{\sigma}}} \\
& =-\frac{2}{3} v \frac{y_{1}^{\prime} y_{2}^{\prime}}{M_{1}^{\prime} M_{5}^{\prime}}+\mathcal{O}\left(\frac{v v_{\sigma}^{2}}{M_{1}^{\prime 2} M_{5}^{\prime 2}}\right)
\end{aligned}
$$

where it has been assumed that $m_{b} \ll m_{t}, m_{\sigma}$ while $m_{\sigma} \ll m_{i}$ for all the other heavy quarks.

Finally, the physical $h$ and $\sigma$ decay widths into two photons are given by

$$
\begin{aligned}
& \Gamma(h \rightarrow \gamma \gamma)=\frac{\alpha^{2} m_{h}^{3}}{64 \pi^{3}}\left|\cos \gamma\left[\Omega_{\hat{h}}^{W}\left(m_{h}^{2}\right)+\Omega_{\hat{h}}^{F}\left(m_{h}^{2}\right)\right]-\sin \gamma \Omega_{\hat{\sigma}}^{F}\left(m_{h}^{2}\right)\right|^{2}, \\
& \Gamma(\sigma \rightarrow \gamma \gamma)=\frac{\alpha^{2} m_{\sigma}^{3}}{64 \pi^{3}}\left|\sin \gamma\left[\Omega_{\hat{h}}^{W}\left(m_{\sigma}^{2}\right)+\Omega_{\hat{h}}^{F}\left(m_{\sigma}^{2}\right)\right]+\cos \gamma \Omega_{\hat{\sigma}}^{F}\left(m_{\sigma}^{2}\right)\right|^{2} .
\end{aligned}
$$

Quantitatively, $\sigma \rightarrow \gamma \gamma$ transitions are dominated by the $W^{ \pm}$loop contributions unless the scalar mixing is small enough for the heavy partner loop contribution to be significant. 


\section{The $\sigma$ resonance at the $\mathrm{LHC}$}

May a light $\sigma$ resonance be lurking in LHC data? In that case, what distinguishes the phenomenology expected for an approximate $\mathrm{SO}(5)$ invariant scenario and that for a generic singlet scalar freely added to the SM Lagrangian? What is the parameter space allowed at present and the discovery reach of the next LHC run? In this section we address these questions.

The constraints from electroweak precision tests explored in section 4.2 showed that a scenario with a light $\sigma$ particle tends to diminish the tension with data. On the other side, from the theoretical viewpoint the assumption of a PNGB nature for the Higgs boson within an approximate global $\mathrm{SO}(5)$ symmetry mildly broken by soft terms prefers a sizeable mass for the $\sigma$ particle, see figure 1. The PNGB interpretation implies the existence of a nonzero mixing between $\sigma$ and $h$, specially when considering naturalness as a guideline since $\sin ^{2} \gamma \sim \xi \ll 1$ would require a strong fine-tuning of the theory -see the discussion after eq. (2.13) and eqs. (2.23), (2.24) and (4.1).

As argued in section 2.3, the scalar potential is completely determined by the masses $m_{h}$ and $m_{\sigma}$, the constant $G_{F}$, and the scalar mixing $\sin \gamma$. The conclusions obtained for the linear $\sigma$ model together with generic soft breaking terms are of general validity. The extra ingredients needed to determine the phenomenology of the $\sigma$ particle are its couplings to the vector-like fermions of the theory, which introduce instead significant model-dependence and may have important consequences particularly in the production of this scalar.

In order to estimate the LHC constraints on the model, we recast many LHC searches for scalar resonances into the $\sigma$ parameter space, calculating the production cross section and decays of the $\sigma$ particle. The production of the $\sigma$ particle at the LHC may proceed mainly via two processes, gluon fusion and vector boson fusion (VBF). Gluon fusion usually dominates the production due to the large gluon pdfs. Nevertheless, this conclusion is somewhat model-dependent as the heavy fermion couplings to $\sigma$ may a priori enhance or diminish the cross section. VBF depends essentially on the mixing angle $\gamma$, but it typically yields a lower production cross section than gluon fusion for $m_{\sigma}<\mathcal{O}(1 \mathrm{TeV})$, for which it will have unnoticeable impact in what follows.

Consider then the cross section for $\sigma$ production via gluon fusion $\sigma(g g \rightarrow \sigma)$. To account for higher order corrections to $\Gamma(\sigma \rightarrow g g)$, we will profit from the results in the literature for a heavy SM-like Higgs boson $H^{\prime}$, using the following approximation

$$
\sigma(g g \rightarrow \sigma) \simeq \frac{|A(\sigma \rightarrow g g)|^{2}}{\left|A_{\mathrm{SM}}\left(H^{\prime} \rightarrow g g\right)\right|^{2}} \sigma_{\mathrm{SM}}\left(g g \rightarrow H^{\prime}\right),
$$

where $A\left(\sigma, H^{\prime} \rightarrow g g\right)$ refers to leading order (one loop) amplitudes and $\sigma_{\mathrm{SM}}\left(g g \rightarrow H^{\prime}\right.$ ) is the NNLO standard gluon fusion production cross section given in ref. [44]. For illustrative purposes we discuss next the LHC impact of the $\sigma$ particle in two steps: first an "only scalars" analysis will be considered, to add next to it the effect of the rather model-dependent fermionic sector.

In the only scalars scenario, that is, a case in which the impact of the heavy fermions on gluon fusion is negligible compared to the top contribution, the production amplitude 

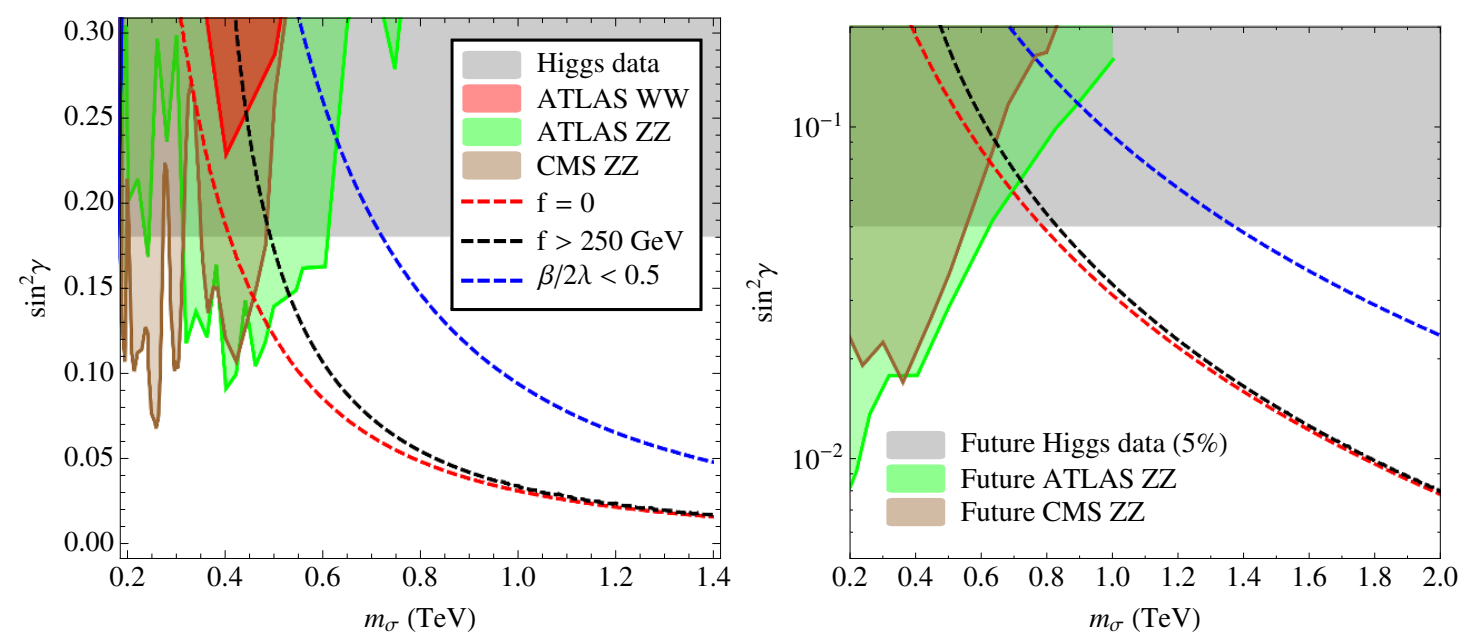

Figure 8. Present LHC (left panel) and future LHC run-2 (right panel) constraints on $\sin ^{2} \gamma$ versus $\sigma$ mass parameter space in the case where gluon fusion is dominated by the top loop. For the latter, a total luminosity of $3 \mathrm{ab}^{-1}$ was assumed.

can be approximated by the top loop contribution for a heavy SM Higgs weighted down by $\sin \gamma$. Under this assumption, we have recasted the LHC searches for a heavy Higgslike particle into constraints in the $\left\{m_{\sigma}, \sin ^{2} \gamma\right\}$ plane, and the results are shown on the left panel of figure 8. The searches taken into account here include diphoton [45, 46], diboson [47-50] and dihiggs $[51,52]$ decays. The figure shows that present LHC data are sensitive to $\sin ^{2} \gamma \simeq 0.1$ for $m_{\sigma}<600 \mathrm{GeV}$, otherwise Higgs measurements put a bound on $\sin ^{2} \gamma<0.18$ independently of $m_{\sigma}$. It is worth noting that these bounds apply well beyond the model discussed in this paper: they are valid for any physics scenario in which the role of the Higgs particle is substituted by a Higgs-scalar system with a generic mixing angle $\gamma$, independently of the details of the theory. In addition, by combining the LHC data with theoretically motivated constraints as those mentioned above, interesting bounds can be derived: a PNGB nature for the Higgs boson corresponds to the area to the right of the red curve depicted, see also figure 1, corresponding to the minimal theoretical requirement $f^{2}>0$ for $\mathrm{SO}(5)$ to be spontaneously broken, resulting in the bound $m_{\sigma}>500 \mathrm{GeV}$ in particular from the impact of ATLAS $H_{\text {heavy }} \rightarrow Z Z$ searches. If $f^{2}$ values above the electroweak scale are instead required (black curve) $m_{\sigma}>550 \mathrm{GeV}$ follows. The future prospects for this "only scalars" scenario are depicted on the right panel of figure 8. It shows the future LHC sensitivity in the $Z Z$ decay channel of the $14 \mathrm{TeV}$ LHC run with an integrated luminosity of $3 \mathrm{ab}^{-1}$, for both ATLAS and CMS [53], as well as the mixing disfavoured by Higgs data assuming a $5 \%$ precision on the Higgs couplings to SM particles. In the absence of any beyond the SM signal, future LHC data together with the aforementioned theory constraints could push the limit on the $\sigma$ mass above $900 \mathrm{GeV}-1.4 \mathrm{TeV}$.

The difference between the LHC predictions of the model discussed in this paper and those stemming from extending the SM by a generic scalar singlet (see e.g. ref. [54]) is the 

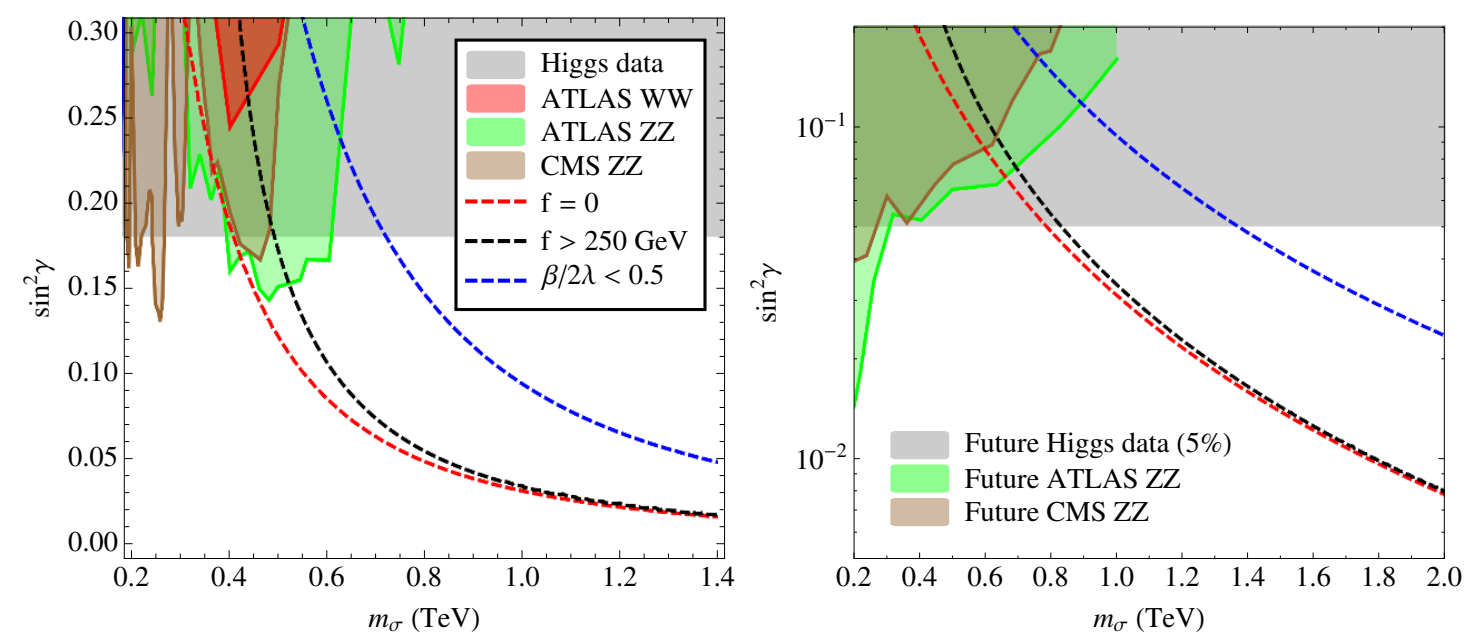

Figure 9. Same as figure 8 , but considering a sizeable contribution of the heavy fermion sector to gluon fusion. See text for details.

underlying $\mathrm{SO}(5)$ structure of the former, which prescribes a specific relation between the quartic terms in the potential as well as specific soft breaking terms. In the generic extra singlet scenarios, the allowed parameter space is given by the entire white area in figure 8 , while a PNGB nature for the Higgs restricts the allowed region to the area to the right of the curves depicted in the figure.

Finally, the impact of the heavy fermions of the model on the gluon fusion cross section may be significant. Using the approximate expressions in eqs. (4.43) and (4.45), assuming that the factor $y_{1} y_{2} / M_{1} M_{5}$ in eqs. (4.40) and (4.41) is the largest contribution between $1 /(4 \pi f)^{2}$ and $1 \mathrm{TeV}^{-2}$ (the latter will also be assumed when $f^{2}<0$ ), the results obtained are depicted in figure 9. It shows that the present LHC bounds on $\sin ^{2} \gamma$ can be weakened by $\mathcal{O}(30-50 \%)$ with respect to the "only scalars" bounds in figure 8 . This is due to a destructive interference between the heavy fermions and the top loops, for the set of parameters considered. Moreover, future searches will be much more sensitive to the heavy fermion sector as they probe smaller mixing angles, and therefore they enter regions in parameter space where the top quark is relatively less important to the $\sigma$ phenomenology.

The $750 \mathrm{GeV}$ di-photon excess. It is a natural question whether the mild $750 \mathrm{GeV}$ di-photon excess observed by ATLAS [55] and CMS [56] could be explained by the $\sigma$ resonance under discussion. This is highly unlikely because of the constraints imposed on the scalar couplings by the approximate $\mathrm{SO}(5)$ symmetry of the scalar potential, as well as the uniqueness of the signal, as explained next.

Since the decay $\sigma \rightarrow \gamma \gamma$ is loop induced, the corresponding branching ratio tends to be very small. In order to be able to account for the excess observed, the $h-\sigma$ mixing needs to be tiny, so that for instance the $W W$ and $Z Z$ channels are suppressed and the loopinduced processes may dominate the decay. This requires $\sin ^{2} \gamma \ll\left(m_{h} / m_{\sigma}\right)^{2} \simeq 0.03$, which eq. (2.23) shows to require on one side $f^{2}<0$ - for which the Higgs cannot be interpreted as a PNGB, and on the other a very small and fine-tuned $\alpha$ value as $\alpha^{2} \propto \beta^{2} \sin ^{2}(2 \gamma)$; overall a very unnatural scenario. 
Furthermore, since the mixing is so small, the top loop essentially does not contribute to the production and decay anymore. Hence, to obtain a large enough $\Gamma(\sigma \rightarrow \gamma \gamma)$ and $\Gamma(\sigma \rightarrow g g)$ the fermion content needs a higher multiplicity than what is assumed in this paper, as well as large Yukawa couplings, since $\sigma$ mainly couples to $T_{1}$ and $T_{5}$ with electric charges $2 / 3$. Even assuming such an extreme and extended configuration, the stability of the potential could become a very serious issue as the fermion contribution to the beta function of the quartic couplings is negative. Additional field content would then be possibly required to compensate for this effect, making the model extremely ad hoc. Therefore, we find no compelling argument to interpret the $750 \mathrm{GeV}$ excess as corresponding to the $\sigma$ scalar studied here.

\section{$6 \quad d \leq 6$ fermionic effective Lagrangian}

The linear model described is renormalizable and valid for any mass range of the fermionic and/or scalar exotic fields. Two simplifying limits are specially interesting: i) the heavy fermion regime, $M \gg m_{\sigma} \gg v$, where $M$ generically represents the exotic fermionic scales $M_{i}$ in eq. (3.3); ii) the heavy singlet regime, $m_{\sigma} \gg M \gg v$. We have concentrated in this paper on the first scenario, considering a not-so-heavy extra singlet in the spectrum and its phenomenological consequences. The second limit is instead interesting to elucidate the connection between the linear (weak) and non-linear (strong) BSM physics scenarios: the $m_{\sigma} \rightarrow \infty$ regime should lead to the non-linear scenarios usually explored in the literature about composite Higgs; it will be discussed in detail in a subsequent publication [27].

When condition i) is satisfied, some important low-energy effects (and model dependencies) induced by the exotic fermions are easily inferred by integrating them out. The procedure is quite lengthy; here only the resulting mass-dimension $(d) 4,5$ and 6 effective operators and their coefficients are summarized. For energies $E<M$, the effective Lagrangian describing the $d \leq 6$ interactions of fermions with gauge and scalar fields can be written as

$$
\mathcal{L}_{\mathrm{eff}}=\bar{q}_{L} i \not D q_{L}+\bar{t}_{R} i \not D t_{R}+\bar{b}_{R} i \not D b_{R}+\sum_{i} c_{i} \mathcal{O}_{i}
$$

where the set $\left\{\mathcal{O}_{i}\right\}$ includes operators of dimension four (for which the induced coefficients are the leading contributions to the top and bottom Yukawa couplings), five and six. We will use the "Warsaw basis" [57] below.

In most models (for instance in composite Higgs ones) it is reasonable to assume that the goldstone boson scale $f$ and the scalar vevs all satisfy $f, v, v_{\sigma} \ll M$. In what follows we will thus assume $f / M \ll 1$ for simplicity, while $\Lambda \approx M$ will be considered with $\Lambda$ denoting generically the composite $\Lambda_{i}$ scales in eq. (3.3). The light field kinetic energies get contributions which require wave function renormalization in order to recover canonically normalized kinetic energies,

$$
\begin{aligned}
q_{L} & \rightarrow \mathcal{Z}_{q_{L}}^{-1 / 2} q_{L} \\
q_{R} & \rightarrow\left(\begin{array}{cc}
\mathcal{Z}_{t_{R}}^{-1 / 2} & 0 \\
0 & \mathcal{Z}_{b_{R}}^{-1 / 2}
\end{array}\right)\left(\begin{array}{l}
t_{R} \\
b_{R}
\end{array}\right),
\end{aligned}
$$




\begin{tabular}{|c|c|}
\hline Coefficient & Leading Order in $f / M$ \\
\hline $\mathcal{Z}_{q_{L}}$ & $\left(1+\frac{\Lambda_{1}^{2}}{M_{5}^{2}}+\frac{\Lambda_{1}^{\prime 2}}{M_{5}^{\prime 2}}\right)$ \\
\hline $\mathcal{Z}_{t_{R}}$ & $\left(1+\frac{\Lambda_{2}^{2}}{M_{5}^{2}}+\frac{\Lambda_{3}^{2}}{M_{1}^{2}}\right)$ \\
\hline $\mathcal{Z}_{b_{R}}$ & $\left(1+\frac{\Lambda_{2}^{\prime 2}}{M_{5}^{\prime 2}}+\frac{\Lambda_{3}^{\prime 2}}{M_{1}^{\prime 2}}\right)$ \\
\hline
\end{tabular}

Table 2. Table with the definitions for the renormalization factors.

where $\mathcal{Z}_{t_{R}}^{-1 / 2}$ and $\mathcal{Z}_{b_{R}}^{-1 / 2}$ are given in table 2 . The operators obtained and their coefficients resulting after those redefinitions, at leading order in $f / M$, are shown in table 3 , where the following definitions have been used,

$$
\begin{aligned}
& \left(H^{\dagger} i \overleftrightarrow{D}_{\mu} H\right) \equiv i\left(H^{\dagger}\left(\vec{D}_{\mu} H\right)-\left(H^{\dagger} \overleftarrow{D}_{\mu}\right) H\right) \\
& \left(H^{\dagger} i \overleftrightarrow{D}_{\mu}^{i} H\right) \equiv i\left(H^{\dagger} \tau^{i}\left(\vec{D}_{\mu} H\right)-\left(H^{\dagger} \overleftarrow{D}_{\mu}\right) \tau^{i} H\right)
\end{aligned}
$$

In writing eq. (6.1) and table 3 the unshifted scalar fields have been assumed. This fact introduces a potential subtlety that we discuss next. Consider for instance the top and bottom quark masses corresponding to the first two operators in the table, which are their respective Yukawa couplings: when the Higgs gets a vev, mass terms for the light quarks are generated. Additional contributions to the light quark masses stem however from the next six operators in the list, for $\sigma=\langle\sigma\rangle$ and $H=\langle H\rangle$. The corrections induced in the top and bottom mass are of higher order in $f / M$, though, and do not need to be retained when working at leading order. Finally,

$$
\begin{aligned}
& m_{t}=\frac{v}{\sqrt{2}}\left(\frac{y_{1} \Lambda_{1} \Lambda_{3}}{M_{1} M_{5}}\right) \frac{1}{\sqrt{\left(1+\frac{\Lambda_{1}^{2}}{M_{5}^{2}}+\frac{\Lambda_{1}^{\prime 2}}{M_{5}^{\prime 2}}\right)\left(1+\frac{\Lambda_{2}^{2}}{M_{5}^{2}}+\frac{\Lambda_{3}^{2}}{M_{1}^{2}}\right)}}\left(1+\mathcal{O}\left(\frac{f}{M}\right)\right), \\
& m_{b}=\frac{v}{\sqrt{2}}\left(\frac{y_{1}^{\prime} \Lambda_{1}^{\prime} \Lambda_{3}^{\prime}}{M_{1}^{\prime} M_{5}^{\prime}}\right) \frac{1}{\sqrt{\left(1+\frac{\Lambda_{1}^{2}}{M_{5}^{2}}+\frac{\Lambda_{1}^{\prime 2}}{M^{\prime 2}}\right)\left(1+\frac{\Lambda_{2}^{\prime 2}}{M_{5}^{\prime 2}}+\frac{\Lambda_{3}^{\prime 2}}{M_{1}^{\prime 2}}\right)}}\left(1+\mathcal{O}\left(\frac{f}{M}\right)\right) .
\end{aligned}
$$

The same reasoning applies to other couplings. For example the fermion- $\sigma$ coupling via the $\mathcal{O}_{\sigma 1}^{t}$ operator [57] would get corrections proportional to $c_{\sigma 2}^{t}\langle\sigma\rangle-$ see table 3 - which are of higher order in the $f / M$ expansion, and can thus be disregarded when restraining to the leading contributions.

\section{Conclusions}

A composite Higgs would manifest with deviations of the Higgs couplings to fermions and gauge vector bosons from the SM predictions. Current data about the Higgs properties are in good agreement with the SM, but the present experimental precision still allow for deviations at the level of $20 \%$ or more, while many channels predicted by the SM have not yet been tested. 


\begin{tabular}{|c|c|c|c|}
\hline & Operator & $c_{i}$ & Leading Order in $f / M$ \\
\hline \multirow{2}{*}{$\operatorname{dim} 4$} & $\bar{q}_{L} \widetilde{H} t_{R}$ & $-y_{t}$ & $-\left(\frac{y_{1} \Lambda_{1} \Lambda_{3}}{M_{1} M_{5}}\right) \mathcal{Z}_{q_{L}}^{-1 / 2} \mathcal{Z}_{t_{R}}^{-1 / 2}$ \\
\hline & $\bar{q}_{L} H b_{R}$ & $-y_{b}$ & $-\left(\frac{y_{1}^{\prime} \Lambda_{1}^{\prime} \Lambda_{3}^{\prime}}{M_{1}^{\prime} M_{5}^{\prime}}\right) \mathcal{Z}_{q_{L}}^{-1 / 2} \mathcal{Z}_{b_{R}}^{-1 / 2}$ \\
\hline \multirow{2}{*}{$\operatorname{dim} 5$} & $\sigma\left(\bar{q}_{L} \widetilde{H} t_{R}\right)$ & $c_{\sigma 1}^{t}$ & $\frac{y_{t}}{M_{5}}\left(y_{2} \frac{\Lambda_{2}}{\Lambda_{3}}-\left(y_{1} \frac{\Lambda_{2} \Lambda_{3}}{M_{1} M_{5}}+y_{2} \frac{\Lambda_{2} \Lambda_{3}}{M_{1}^{2}}\right) \mathcal{Z}_{t_{R}}^{-1}\right)$ \\
\hline & $\sigma\left(\bar{q}_{L} H b_{R}\right)$ & $c_{\sigma 1}^{b}$ & $\frac{y_{b}}{M_{5}^{\prime}}\left(y_{2}^{\prime} \frac{\Lambda_{2}^{\prime}}{\Lambda_{3}^{\prime}}-\left(y_{1}^{\prime} \frac{\Lambda_{2}^{\prime} \Lambda_{3}^{\prime}}{M_{1}^{\prime} M_{5}^{\prime}}+y_{2}^{\prime} \frac{\Lambda_{2}^{\prime} \Lambda_{3}^{\prime}}{M_{1}^{\prime 2}}\right) \mathcal{Z}_{b_{R}}^{-1}\right)$ \\
\hline \multirow{9}{*}{$\operatorname{dim} 6$} & $\sigma^{2}\left(\bar{q}_{L} \widetilde{H} t_{R}\right)$ & $c_{\sigma 2}^{t}$ & $\begin{aligned}-\frac{y_{t}}{M_{1} M_{5}}\left(y_{1} y_{2}-\right. & \left(y_{1} y_{2}\left(2 \frac{\Lambda_{2}^{2}}{M_{5}^{2}}+\frac{\Lambda_{3}^{2}}{M_{1}^{2}}\right)+\frac{3 y_{2}^{2} \Lambda_{2}^{2}+y_{1}^{2} \Lambda_{3}^{2}}{2 M_{1} M_{5}}\right) \mathcal{Z}_{t_{R}}^{-1} \\
& \left.+2 \frac{\Lambda_{2}^{2} \Lambda_{3}^{2}}{M_{1} M_{5}}\left(\frac{y_{1}^{2}}{M_{5}^{2}}+\frac{2 y_{1} y_{2}}{M_{5} M_{1}}+\frac{y_{2}^{2}}{M_{1}^{2}}\right) \mathcal{Z}_{t_{R}}^{-2}\right)\end{aligned}$ \\
\hline & $\sigma^{2}\left(\bar{q}_{L} H b_{R}\right)$ & $c_{\sigma 2}^{b}$ & $\begin{array}{c}-\frac{y_{b}}{M_{1}^{\prime} M_{5}^{\prime}}\left(y_{1}^{\prime} y_{2}^{\prime}-\left(y_{1}^{\prime} y_{2}^{\prime}\left(2 \frac{\Lambda_{2}^{\prime 2}}{M^{\prime 2}}+\frac{\Lambda_{3}^{\prime 2}}{M^{\prime 2}}\right)+\frac{3 y_{2}^{\prime 2} \Lambda_{2}^{\prime 2}+y_{1}^{\prime 2} \Lambda_{3}^{\prime 2}}{2 M_{1}^{\prime} M_{5}^{\prime}}\right) \mathcal{Z}_{b_{R}}^{-1}\right. \\
\left.+2 \frac{\Lambda_{2}^{\prime 2} \Lambda_{3}^{\prime 2}}{M_{1}^{\prime} M_{5}^{\prime}}\left(\frac{y_{1}^{\prime 2}}{M^{\prime 2}}+\frac{2 y_{1}^{\prime} y_{2}^{\prime}}{M_{5}^{\prime} M_{1}^{\prime}}+\frac{y_{2}^{\prime 2}}{M_{1}^{\prime 2}}\right)^{-1} \mathcal{Z}_{b_{R}}^{-2}\right)\end{array}$ \\
\hline & $|H|^{2}\left(\bar{q}_{L} \widetilde{H} t_{R}\right)$ & $c_{H 2}^{t}$ & $\begin{aligned} &-\frac{y_{t}}{M_{1} M_{5}}\left(2 y_{1} y_{2}-\right.\left(2 y_{1} y_{2} \frac{\Lambda_{3}^{2}}{M_{1}^{2}}+y_{1}^{2} \frac{\Lambda_{3}^{2}}{M_{1} M_{5}}\right) \mathcal{Z}_{t_{R}}^{-1} \\
&\left.-\left(y_{1} y_{2} \frac{\Lambda_{1}^{2}}{M_{5}^{2}}+\frac{y_{1}^{2}}{2} \frac{\Lambda_{1}^{2}}{M_{1} M_{5}}\right) \mathcal{Z}_{q_{L}}^{-1}\right)\end{aligned}$ \\
\hline & $|H|^{2}\left(\bar{q}_{L} H b_{R}\right)$ & $c_{H 2}^{b}$ & $\begin{aligned}-\frac{y_{b}}{M_{1}^{\prime} M_{5}^{\prime}}\left(2 y_{1}^{\prime} y_{2}^{\prime}-(\right. & \left(2 y_{1}^{\prime} y_{2}^{\prime} \frac{\Lambda_{3}^{\prime 2}}{M_{1}^{\prime 2}}+y_{1}^{\prime 2} \frac{\Lambda_{3}^{\prime 2}}{M_{1}^{\prime} M_{5}^{\prime}}\right) \mathcal{Z}_{b_{R}}^{-1} \\
& \left.-\left(y_{1}^{\prime} y_{2}^{\prime} \frac{\Lambda_{1}^{\prime 2}}{M_{5}^{\prime 2}}+\frac{y_{1}^{\prime 2}}{2} \frac{\Lambda^{\prime 2}}{M_{1}^{\prime} M_{5}^{\prime}}\right) \mathcal{Z}_{q_{L}}^{-1}\right)\end{aligned}$ \\
\hline & $\left(H^{\dagger} i \overleftrightarrow{D}_{\mu} H\right)\left(\bar{q}_{L} \gamma^{\mu} q_{L}\right)$ & $c_{L}^{(1)}$ & $\frac{1}{4}\left(\frac{y_{1}^{2} \Lambda_{1}^{2}}{M_{1}^{2} M_{5}^{2}}-\frac{y_{1}^{\prime 2} \Lambda_{1}^{\prime 2}}{M_{1}^{\prime 2} M_{5}^{\prime 2}}\right) \mathcal{Z}_{q_{L}}^{-1}$ \\
\hline & $\left(H^{\dagger} i \overleftrightarrow{D}_{\mu}^{i} H\right)\left(\bar{q}_{L} \tau^{i} \gamma^{\mu} q_{L}\right)$ & $c_{L}^{(3)}$ & $-\frac{1}{4}\left(\frac{y_{1}^{2} \Lambda_{1}^{2}}{M_{1}^{2} M_{5}^{2}}+\frac{y_{1}^{\prime 2} \Lambda_{1}^{\prime 2}}{M_{1}^{\prime 2} M^{\prime 2}}\right) \mathcal{Z}_{q_{L}}^{-1}$ \\
\hline & $\left(H^{\dagger} i \overleftrightarrow{D}_{\mu} H\right)\left(\bar{t}_{R} \gamma^{\mu} t_{R}\right)$ & $c_{R}^{t}$ & 0 \\
\hline & $\left(H^{\dagger} i \overleftrightarrow{D}_{\mu} H\right)\left(\bar{b}_{R} \gamma^{\mu} b_{R}\right)$ & $c_{R}^{b}$ & 0 \\
\hline & $i\left(\widetilde{H}^{\dagger} D_{\mu} H\right)\left(\bar{t}_{R} \gamma^{\mu} b_{R}\right)$ & $c_{R}^{t b}$ & 0 \\
\hline
\end{tabular}

Table 3. Leading order low-energy effective operators induced and their coefficients. Note that the Yukawa couplings defined in the two first rows appear as well in coefficients of some higher order operators. The renormalization factors present have been defined in table 2. Those operators made out exclusively of SM fields have been written in the Warsaw basis [57]. 
Completely clarifying the mechanism of electroweak breaking is one of the main goals of particle physics today and an important role, on the theory side, is played by simple and motivated extensions of the SM which could provide guidance in the experimental search. To this purpose we have formulated a model where the scalar sector of the SM is minimally extended to include an additional scalar particle $\sigma$. We are motivated by the attractive possibility that the Higgs itself can be interpreted as a pseudo-Goldstone boson associated to the breaking of an approximate symmetry. The most economic custodial preserving possibility is offered by a global $\mathrm{SO}(5)$ spontaneously broken down to $\mathrm{SO}(4)$, thus generating the four components of the Higgs doublet as Goldstone bosons. Indeed this case has already been vastly analyzed in the literature in the strongly interacting regime, either in the context of four-dimensional models where $\mathrm{SO}(5)$ is nonlinearly realized or in five-dimensional models with a warped space-time metric. The latter, weakly coupled duals to strongly coupled four-dimensional conformal theories, are believed to provide a calculable framework for composite Higgs models.

In our model, where a scalar fiveplet of $\mathrm{SO}(5)$ comprises both an electroweak doublet and an extra singlet $\sigma$, the $\mathrm{SO}(5)$ symmetry is linearly realized and the theory is renormalizable. It could be viewed as the simplest UV completion in the class of models based on the coset $\mathrm{SO}(5) / \mathrm{SO}(4)$. In this way we lose generality, but we gain in calculability and predictability. We can study accurately the regime where the symmetry breaking sector is in the perturbative regime and provide a useful interpolation between the weakly and the strongly interacting cases. The $\mathrm{SO}(5)$ invariant part of the symmetry breaking sector contains just two parameters, the symmetry breaking scale and the mass of the $\sigma$ particle. In the $\mathrm{SO}(5)$ invariant limit the Higgs particle is a true massless Goldston boson. Other two parameters, arising from the one-loop effective potential when gauge and Yukawa interactions are turned on, break the $\mathrm{SO}(5)$ symmetry softly, fix the relative orientation between the residual $\mathrm{SO}(4)$ invariance and the $\mathrm{SU}(2) \times \mathrm{U}(1)$ electroweak group and provide the Higgs boson a mass. A mixing angle $\gamma$ defines the two physical mass eigenstates as mixtures of the electroweak doublet and the singlet.

As the Higgs mass and the value of the electroweak scale are known, the scalar parameter space is thus completely defined in terms of the $\sigma$ mass and $\sin \gamma$. We have identified in it the areas in which the Higgs can be considered a pseudo-Goldstone boson, resulting in two well-differentiated regions corresponding respectively to a $\sigma$ particle lighter and heavier than the Higgs particle. The former case has phenomenological interest, but it turns out to require fine-tuned parameters in the scalar potential and indeed it would call for an explanation of the stability of such a light $\sigma$; in consequence, we have focused most of the analysis on the region in which the $\sigma$ is heavier than the Higgs, to which the remarks that follow apply.

The UV completion of the theory would require further explanation as far as the $\sigma$ particle is light enough for the theory to remain in the perturbative realm, as the model would have then replaced the hierarchy problem for the Higgs mass for that of the $\sigma$ mass. It is nevertheless a most useful tool to explore a dynamical origin for the Higgs and the possibility of new degrees of freedom appearing in foreseen experiments. For heavy $\sigma$ the symmetry breaking sector becomes strongly interacting and in the limit of infinite $m_{\sigma}$ we fall into a nonlinear realization of $\mathrm{SO}(5)$. The couplings of the physical Higgs $h$ to $W$ and 
$Z$ are suppressed by $\cos \gamma$, while the heavier $\sigma$ state couples to $W$ and $Z$ with a strength proportional to $\sin \gamma$. Present data on the Higgs decay into $W W$ require a relatively small mixing between the Higgs field and the new scalar, leaving however a considerable room to a departure from the SM picture. We have also identified the differences in experimental impact of the case of a generic singlet scalar added to the SM and the case of the approximately $\mathrm{SO}(5)$ invariant scalar sector under discussion.

The scalar sector of the non-linear $\mathrm{SO}(5) \sigma$ model developed is minimal, simple and of general validity. A significant model dependence comes in when considering the fermion sector. We chose to describe fermion masses within a simple realization of the partial compositeness idea. We introduced a set of new vector-like heavy fermions, which couple to the full $\mathrm{SO}(5)$ scalar fiveplets. Ordinary fermions do not directly interact with scalars but mix with the heavy fermions. At low energies such a mixing gives rise to the usual Yukawa couplings and fermion masses. To keep our model as minimal as possible, we assign the heavy fermions to singlets and fiveplets. By focusing on the third generation of quarks, we introduce one singlet and one fiveplet per each charge sector. Even in this minimal setup, the fermion sector of the model brings in 14 parameters: four heavy fermion masses, four independent Yukawa couplings and six mixing parameters. For completeness and as first step towards a low-energy benchmark effective Lagrangian, we have separately integrated out the heavy fermions and obtained the ensuing dimension four, five and six effective operators made out of only SM fields or $\sigma$ plus SM fields, which include Yukawa and other couplings for the lighter particles. Nevertheless, all the phenomenological analyses have been performed with dynamical heavy fermions.

We have analyzed precision electroweak observables, globally parametrized in terms of the $S, T$ and $R_{b}$ parameters. Concerning the scalar contribution, from an explicit one-loop computation we recover a well-known result: a positive contribution to the $S$ parameter and a negative contribution to the $T$ parameter. At variance with generic models where $\mathrm{SO}(5)$ is nonlinearly realized, we do not have any cut-off ambiguity. In our model the extra contributions $\Delta S$ and $\Delta T$ are finite. They vanish when $\sin \gamma$ goes to zero, since the scalar $\sigma$ does not couple any more to $W$ and $Z$ in this limit. They also vanish when $h$ and $\sigma$ have the same mass, since in this case the angle $\gamma$ loses any physical meaning. If $h$ and $\sigma$ are close in mass, the contribution to $\Delta S$ and $\Delta T$ is reduced, compared to nonlinear realizations. The main impact of the heavy fermion contribution is on the $T$ parameter. Letting the heavy fermions to be as light as $800 \mathrm{GeV}$, the lower limit from direct searches, contributions to $T$ of both signs as large as 0.2 in magnitude are generated by scanning the parameter space. Positive (negative) $\Delta T$ are correlated to a sizable mixing between $t_{L}\left(t_{R}\right)$ and an electroweak singlet (doublet) heavy fermion component. Fermionic contributions to $S$ are typically positive and smaller, while those to $R_{b}$ are mainly negative and of order few per mil. Even the largest scalar contributions to $\Delta S$ and $\Delta T$, obtained when $\sin ^{2} \gamma$ saturates its experimental bound and the scalar $\sigma$ is very heavy, can always be compensated by the fermionic ones for an appropriate choice of the parameters, thus keeping $S$ and $T$ within the experimentally allowed region.

The Higgs production at LHC proceeds mainly via gluon fusion as in the SM. The amplitude comprises the usual SM contribution weighted by $\cos \gamma$ and an extra contribution proportional to $\sin \gamma$ which decouples in the heavy fermion limit. The interference between 
the two can be both constructive or destructive. The effective coupling of the Higgs to a diphoton pair is modified in a similar way. In the large mass limit for the heavy fermions - taking the limit in such a way that the Higgs and light masses remain finite and at their physical values - their impact can be neglected and deviations from the SM only depend on $\sin \gamma$. In particular the Higgs decay to $W W$ allows to put the bound $\sin ^{2} \gamma<0.18$, at $2 \sigma$.

The production of $\sigma$ at the LHC can mainly proceed via vector boson fusion or gluon fusion, the latter typically dominating for a $\sigma$ mass not exceeding few $\mathrm{TeV}$. There are no direct couplings of $\sigma$ to gluons but, as for the Higgs, an effective coupling arises from fermion loops. In the regime where the heavy fermions decouple the effective $\sigma$ coupling to gluons is controlled by the top loop weighted by a $\sin \gamma$ factor. Present direct LHC searches for a scalar particle are already sensitive to $\sin ^{2} \gamma$ of order $0.1-0.2$, for $m_{\sigma}<600 \mathrm{GeV}$. If we combine this result with the theoretical requirement that the Higgs behaves as a pseudoGoldstone boson, we can already exclude $\sigma$ masses below about $500 \mathrm{GeV}$. This limit can be pushed to $900-1400 \mathrm{GeV}$ by future LHC data from run 2 , in the absence of any signal of new physics.

While in principle our model contains the ingredients to explain the recently observed $750 \mathrm{GeV}$ diphoton excess in terms of $\sigma$ production and decay into a photon pair, such an interpretation is rather unnatural since it would involve a tuning of the parameters in the scalar sector and, to boost both production and decay, a heavy fermion multiplicity much larger that the one adopted in the present version. Clearly, together with the modifications of the SM Higgs couplings, the prediction of an additional scalar, potentially observable at the next LHC run, is the distinctive feature of our model. However, due to the mixing with an electroweak doublet, the new particle is not expected to decay exclusively into photons but rather into a variety of channels, much as the SM Higgs does.

\section{Acknowledgments}

It is a pleasure to thank Marcela Carena, Tony Gherghetta, Oleksii Matsedonskyi and Luca Merlo for useful discussions. The authors (each identified by the first letter of her/his last name) acknowledge partial financial support by the European Union through the FP7 ITN INVISIBLES (PITN-GA-2011-289442) (FGKMRS), by the Horizon2020 RISE InvisiblesPlus 690575 (FGKMRS), by CiCYT through the project FPA2012-31880 (GS), and by the Spanish MINECO through the Centro de excelencia Severo Ochoa Program under grant SEV-2012-0249 (GMS). The work of K.K. is supported by an ESR contract of the European Union network FP7 ITN INVISIBLES mentioned above. The work of S.S. is supported through the grant BES-2013-066480 of the Spanish MICINN and she also received support during from the Spanish MICINN grant EEBB-I-15-10242. We would also like to thank the Kavli Institute for Theoretical Physics - Santa Barbara (GM) (National Science Foundation grant PHY11-25915), the Mainz Institute for Theoretical Physics (M), the Physics Department of the University of California San Diego (S), the Aspen Center for Physics (GM) (National Science Foundation grant PHY-1066293 and the Simons Foundation) and the Institute for Theoretical Physics IFT-UAM/CSIC (F) for hospitality and/or partial support during the completion of this work. 


\section{A Coleman-Weinberg potential}

In section 2.1 it was assumed a specific form for the $\mathrm{SO}(5)$ scalar potential broken to $\mathrm{SO}(4)$, introducing two additional $\mathrm{SO}(5)$ breaking parameters $\alpha$ and $\beta$. In this section we will further motivate this assumption. Even assuming that the tree level scalar potential would preserve the global $\mathrm{SO}(5)$ symmetry, the presence of a $\mathrm{SO}(5)$ breaking couplings in the fermionic sector will generate at one-loop level $\mathrm{SO}(5)$ breaking terms through the Coleman-Weinberg mechanism [58]. The one-loop fermionic contribution can be obtained from the field dependent mass matrix $\mathcal{M}$ as

$$
\begin{aligned}
V_{\text {loop }}=-\frac{i}{2} \int \frac{d^{4} k}{(2 \pi)^{4}} \sum_{n=1}^{\infty} \frac{1}{n} \operatorname{Tr}\left(\frac{\mathcal{M M}^{\dagger}}{k^{2}}\right)^{n}=\frac{i}{2} \int \frac{d^{4} k}{(2 \pi)^{4}} \operatorname{Tr} \log \left(1-\frac{\mathcal{M M}^{\dagger}}{k^{2}}\right) \\
=-\frac{1}{64 \pi^{2}}\left(\Lambda^{2} \operatorname{Tr}\left[\mathcal{M} \mathcal{M}^{\dagger}\right]-\operatorname{Tr}\left[\left(\mathcal{M} \mathcal{M}^{\dagger}\right)^{2}\right] \log \left(\frac{\Lambda^{2}}{\mu^{2}}\right)\right. \\
\left.+\operatorname{Tr}\left[\left(\mathcal{M} \mathcal{M}^{\dagger}\right)^{2} \log \left(\frac{\mathcal{M M}^{\dagger}}{\mu^{2}}\right)\right]-\frac{1}{2} \operatorname{Tr}\left[\left(\mathcal{M M}^{\dagger}\right)^{2}\right]\right),
\end{aligned}
$$

where $\Lambda$ is the UV cutoff scale while $\mu$ is a generic renormalization scale. The first two terms on the right-hand side of this equation are divergent, respectively quadratically and logarithmically, while the last two terms are finite. For the model under discussion it results:

$$
\begin{aligned}
\operatorname{Tr}\left[\mathcal{M M}^{\dagger}\right] & =c_{1}+c_{2}\left(\phi^{T} \phi\right), \\
\operatorname{Tr}\left[\left(\mathcal{M M}^{\dagger}\right)^{2}\right] & =d_{1}+d_{2} \sigma+d_{3} \sigma^{2}+d_{4}\left(\phi^{T} \phi\right)+d_{5}\left(\phi^{T} \phi\right)^{2},
\end{aligned}
$$

where

$$
\begin{aligned}
& c_{1}=2 \Lambda_{1}^{2}+\Lambda_{2}^{2}+\Lambda_{3}^{2}+M_{1}^{2}+5 M_{5}^{2}+\left(\{\} \leftrightarrow\{\}^{\prime}\right), \\
& c_{2}=y_{1}^{2}+y_{2}^{2}+\left(\{\} \leftrightarrow\{\}^{\prime}\right),
\end{aligned}
$$

and

$$
\begin{aligned}
& d_{1}=M_{1}^{4}+5 M_{5}^{4}+2 M_{5}^{2}\left(2 \Lambda_{1}^{2}+\Lambda_{2}^{2}\right)+2 M_{1}^{2} \Lambda_{3}^{2}+2 \Lambda_{1}^{4}+\left(\Lambda_{2}^{2}+\Lambda_{3}^{2}\right)^{2}+\left(\{\} \leftrightarrow\{\}^{\prime}\right), \\
& d_{2}=4\left(y_{1} M_{1}+y_{2} M_{5}\right) \Lambda_{2} \Lambda_{3}+\left(\{\} \leftrightarrow\{\}^{\prime}\right), \\
& d_{3}=2 y_{1}^{2} \Lambda_{2}^{2}-y_{2}^{2} \Lambda_{1}^{2}+\left(\{\} \leftrightarrow\{\}^{\prime}\right), \\
& d_{4}=4 y_{1} y_{2} M_{1} M_{5}+2\left(y_{1}^{2}+y_{2}^{2}\right)\left(M_{1}^{2}+M_{5}^{2}\right)+y_{2}^{2}\left(\Lambda_{1}^{2}+2 \Lambda_{3}^{2}\right)+\left(\{\} \leftrightarrow\{\}^{\prime}\right), \\
& d_{5}=y_{1}^{4}+y_{2}^{4}+\left(\{\} \leftrightarrow\{\}^{\prime}\right) .
\end{aligned}
$$

In consequence, only the quadratically divergent piece is seen to remain $\mathrm{SO}(5)$ invariant, while the rest of the potential introduces an explicit breaking of the $\mathrm{SO}(5)$ symmetry to $\mathrm{SO}(4)$, see eq. (A.3). The quadratic divergence can be thus absorbed in the parameters of the tree-level Lagrangian, and the same holds for the $\mathrm{SO}(5)$ invariant component of the logarithmically divergent terms $\left(d_{1}, d_{4}\right.$ and $\left.d_{5}\right)$. However, the presence of the $d_{2}$ and 
$d_{3}$ divergent $\mathrm{SO}(5)$-breaking terms require to add the two corresponding counterterms in the potential, so as to obtain a renormalizable theory. These two necessary terms are those defined with coefficients $\alpha$ and $\beta$ in the potential definition eq. (2.9). The gauge couplings appearing in the covariant derivatives also break explicitly the $\mathrm{SO}(5)$ symmetry, but they do not induce extra one-loop divergent contributions to the effective potential, and in consequence only $\alpha$ and $\beta$ are required for consistency.

The computation of the finite part of $V_{\text {loop }}$ should provide the dependence of the parameters on the renormalization scale ${ }^{13}$ and should thus be equivalent to the computation of their renormalization group equations; this task is beyond the scope of the present paper.

Open Access. This article is distributed under the terms of the Creative Commons Attribution License (CC-BY 4.0), which permits any use, distribution and reproduction in any medium, provided the original author(s) and source are credited.

\section{References}

[1] S.L. Glashow, J. Iliopoulos and L. Maiani, Weak Interactions with Lepton-Hadron Symmetry, Phys. Rev. D 2 (1970) 1285 [INSPIRE].

[2] M.K. Gaillard, B.W. Lee and J.L. Rosner, Search for Charm, Rev. Mod. Phys. 47 (1975) 277 [INSPIRE].

[3] M.E. Peskin, The Alignment of the Vacuum in Theories of Technicolor, Nucl. Phys. B 175 (1980) 197 [INSPIRE].

[4] J. Preskill, Subgroup Alignment in Hypercolor Theories, Nucl. Phys. B 177 (1981) 21 [INSPIRE].

[5] D.B. Kaplan and H. Georgi, SU(2) $\times$ U(1) Breaking by Vacuum Misalignment, Phys. Lett. B 136 (1984) 183 [INSPIRE].

[6] H. Georgi and D.B. Kaplan, Composite Higgs and Custodial SU(2), Phys. Lett. B 145 (1984) 216 [INSPIRE].

[7] M.J. Dugan, H. Georgi and D.B. Kaplan, Anatomy of a Composite Higgs Model, Nucl. Phys. B 254 (1985) 299 [INSPIRE].

[8] K. Agashe, R. Contino and A. Pomarol, The Minimal composite Higgs model, Nucl. Phys. B 719 (2005) 165 [hep-ph/0412089] [INSPIRE].

[9] R. Contino, L. Da Rold and A. Pomarol, Light custodians in natural composite Higgs models, Phys. Rev. D 75 (2007) 055014 [hep-ph/0612048] [InSPIRE].

[10] A. Manohar and H. Georgi, Chiral Quarks and the Nonrelativistic Quark Model, Nucl. Phys. B 234 (1984) 189 [INSPIRE].

[11] D.B. Kaplan, Flavor at SSC energies: A New mechanism for dynamically generated fermion masses, Nucl. Phys. B 365 (1991) 259 [InSPIRE].

\footnotetext{
${ }^{13}$ The gauge bosons induce finite contributions which are usually neglected with respect to the fermionic ones, as their relative ratio is proportional to the ratio between the mass scale for the heavy fermions and that for the gauge bosons.
} 
[12] ATLAS collaboration, Search for production of vector-like quark pairs and of four top quarks in the lepton-plus-jets final state in pp collisions at $\sqrt{s}=8 \mathrm{TeV}$ with the ATLAS detector, JHEP 08 (2015) 105 [arXiv: 1505.04306] [INSPIRE].

[13] CMS collaboration, Search for top quark partners with charge $5 / 3$ at $\sqrt{s}=13 \mathrm{TeV}$, CMS-PAS-B2G-15-006.

[14] G. Panico, M. Redi, A. Tesi and A. Wulzer, On the Tuning and the Mass of the Composite Higgs, JHEP 03 (2013) 051 [arXiv:1210.7114] [INSPIRE].

[15] M. Carena, L. Da Rold and E. Pontón, Minimal Composite Higgs Models at the LHC, JHEP 06 (2014) 159 [arXiv: 1402.2987] [INSPIRE].

[16] R. Contino, D. Marzocca, D. Pappadopulo and R. Rattazzi, On the effect of resonances in composite Higgs phenomenology, JHEP 10 (2011) 081 [arXiv:1109.1570] [INSPIRE].

[17] D. Marzocca, M. Serone and J. Shu, General Composite Higgs Models, JHEP 08 (2012) 013 [arXiv: 1205.0770] [INSPIRE].

[18] M. Redi and A. Tesi, Implications of a Light Higgs in Composite Models, JHEP 10 (2012) 166 [arXiv: 1205.0232] [inSPIRE].

[19] A. Carmona and F. Goertz, A naturally light Higgs without light Top Partners, JHEP 05 (2015) 002 [arXiv:1410.8555] [INSPIRE].

[20] G. von Gersdorff, E. Pontón and R. Rosenfeld, The Dynamical Composite Higgs, JHEP 06 (2015) 119 [arXiv: 1502.07340] [inSPIRE].

[21] M. Gell-Mann and M. Levy, The axial vector current in beta decay, Nuovo Cim. 16 (1960) 705 [INSPIRE].

[22] R. Barbieri, B. Bellazzini, V.S. Rychkov and A. Varagnolo, The Higgs boson from an extended symmetry, Phys. Rev. D 76 (2007) 115008 [arXiv:0706.0432] [INSPIRE].

[23] T. Alanne, H. Gertov, F. Sannino and K. Tuominen, Elementary Goldstone Higgs boson and dark matter, Phys. Rev. D 91 (2015) 095021 [arXiv:1411.6132] [InSPIRE].

[24] H. Gertov, A. Meroni, E. Molinaro and F. Sannino, Theory and phenomenology of the elementary Goldstone Higgs boson, Phys. Rev. D 92 (2015) 095003 [arXiv:1507.06666] [INSPIRE].

[25] R. Alonso, M.B. Gavela, L. Merlo, S. Rigolin and J. Yepes, The Effective Chiral Lagrangian for a Light Dynamical "Higgs Particle", Phys. Lett. B 722 (2013) 330 [Erratum ibid. B 726 (2013) 926] [arXiv: 1212.3305] [INSPIRE].

[26] G. Buchalla, O. Catà and C. Krause, Complete Electroweak Chiral Lagrangian with a Light Higgs at NLO, Nucl. Phys. B 880 (2014) 552 [arXiv:1307.5017] [INSPIRE].

[27] F. Feruglio et al., in preparation.

[28] L.J. Dixon and Y. Li, Bounding the Higgs Boson Width Through Interferometry, Phys. Rev. Lett. 111 (2013) 111802 [arXiv: 1305.3854] [INSPIRE].

[29] Particle Data Group collaboration, K.A. Olive et al., Review of Particle Physics, Chin. Phys. C 38 (2014) 090001 [INSPIRE].

[30] J.F. Gunion, H.E. Haber, G.L. Kane and S. Dawson, The Higgs Hunter's Guide, Front. Phys. 80 (2000) 1 [INSPIRE]. 
[31] K. Agashe, R. Contino, L. Da Rold and A. Pomarol, A Custodial symmetry for Z $b \bar{b}$, Phys. Lett. B 641 (2006) 62 [hep-ph/0605341] [INSPIRE].

[32] ATLAS, CMS collaborations, Measurements of the Higgs boson production and decay rates and constraints on its couplings from a combined ATLAS and CMS analysis of the LHC pp collision data at $\sqrt{s}=7$ and $8 \mathrm{TeV}$, ATLAS-CONF-2015-044.

[33] G. Panico and A. Wulzer, The Composite Nambu-Goldstone Higgs, Lect. Notes Phys. 913 (2016) pp.1-316 [arXiv:1506.01961] [InSPIRE].

[34] C. Anastasiou, E. Furlan and J. Santiago, Realistic Composite Higgs Models, Phys. Rev. D 79 (2009) 075003 [arXiv:0901.2117] [InSPIRE].

[35] D. Ghosh, M. Salvarezza and F. Senia, Extending the Analysis of Electroweak Precision Constraints in Composite Higgs Models, arXiv:1511.08235 [INSPIRE].

[36] M.E. Peskin and T. Takeuchi, Estimation of oblique electroweak corrections, Phys. Rev. D 46 (1992) 381 [INSPIRE].

[37] G. Altarelli and R. Barbieri, Vacuum polarization effects of new physics on electroweak processes, Phys. Lett. B 253 (1991) 161 [INSPIRE].

[38] M. Ciuchini, E. Franco, S. Mishima, M. Pierini, L. Reina and L. Silvestrini, Update of the electroweak precision fit, interplay with Higgs-boson signal strengths and model-independent constraints on new physics, in International Conference on High Energy Physics 2014 (ICHEP 2014), Valencia, Spain, 2-9 July 2014 [arXiv: 1410.6940] [INSPIRE].

[39] V.A. Novikov, L.B. Okun and M.I. Vysotsky, On the Electroweak one loop corrections, Nucl. Phys. B 397 (1993) 35 [INSPIRE].

[40] A. Orgogozo and S. Rychkov, The S parameter for a Light Composite Higgs: a Dispersion Relation Approach, JHEP 06 (2013) 014 [arXiv:1211.5543] [INSPIRE].

[41] H.E. Haber and D. O'Neil, Basis-independent methods for the two-Higgs-doublet model III: The CP-conserving limit, custodial symmetry and the oblique parameters $S, T, U$, Phys. Rev. D 83 (2011) 055017 [arXiv: 1011.6188] [INSPIRE].

[42] L. Lavoura and J.P. Silva, The Oblique corrections from vector-like singlet and doublet quarks, Phys. Rev. D 47 (1993) 2046 [InSPIRE].

[43] S. Dawson and E. Furlan, A Higgs Conundrum with Vector Fermions, Phys. Rev. D 86 (2012) 015021 [arXiv: 1205.4733] [INSPIRE].

[44] LHC Higgs Cross Section Working Group collaboration, J.R. Andersen et al., Handbook of LHC Higgs Cross Sections: 3. Higgs Properties, arXiv:1307.1347 [InSPIRE].

[45] ATLAS collaboration, Search for Scalar Diphoton Resonances in the Mass Range 65-600 GeV with the ATLAS Detector in pp Collision Data at $\sqrt{s}=8 \mathrm{TeV}$, Phys. Rev. Lett. 113 (2014) 171801 [arXiv:1407.6583] [INSPIRE].

[46] CMS collaboration, Search for diphoton resonances in the mass range from 150 to $850 \mathrm{GeV}$ in pp collisions at $\sqrt{s}=8 \mathrm{TeV}$, Phys. Lett. B 750 (2015) 494 [arXiv:1506.02301] [INSPIRE].

[47] ATLAS collaboration, Search for a high-mass Higgs boson decaying to a $W$ boson pair in $p p$ collisions at $\sqrt{s}=8 \mathrm{TeV}$ with the ATLAS detector, JHEP 01 (2016) 032 [arXiv: 1509.00389] [INSPIRE].

[48] ATLAS collaboration, Search for an additional, heavy Higgs boson in the $H \rightarrow Z Z$ decay channel at $\sqrt{s}=8 \mathrm{TeV}$ in $p p$ collision data with the ATLAS detector, Eur. Phys. J. C 76 (2016) 45 [arXiv: 1507.05930] [INSPIRE]. 
[49] CMS collaboration, Properties of the Higgs-like boson in the decay $H \rightarrow Z Z \rightarrow 4 \ell$ in pp collisions at $\sqrt{s}=7$ and $8 \mathrm{TeV}$, CMS-PAS-HIG-13-002.

[50] CMS collaboration, Update on the search for the standard model Higgs boson in pp collisions at the $\mathrm{LHC}$ decaying to $\mathrm{W}^{+} W^{-}$in the fully leptonic final state, CMS-PAS-HIG-13-003.

[51] CMS collaboration, Search for resonant pair production of Higgs bosons decaying to two bottom quark-antiquark pairs in proton-proton collisions at 8 TeV, Phys. Lett. B 749 (2015) 560 [arXiv: 1503.04114] [INSPIRE].

[52] ATLAS collaboration, Searches for Higgs boson pair production in the $h h \rightarrow b b \tau \tau, \gamma \gamma W W^{*}, \gamma \gamma b b, b b b b$ channels with the ATLAS detector, Phys. Rev. D 92 (2015) 092004 [arXiv: 1509.04670] [INSPIRE].

[53] ATLAS, CMS collaborations, A. Holzner, Beyond standard model Higgs physics: prospects for the High Luminosity LHC, arXiv:1411.0322 [INSPIRE].

[54] V. Martín Lozano, J.M. Moreno and C.B. Park, Resonant Higgs boson pair production in the $h h \rightarrow b \bar{b} W W \rightarrow b \bar{b} \ell^{+} \nu \ell^{-} \bar{\nu}$ decay channel, JHEP 08 (2015) 004 [arXiv:1501.03799] [INSPIRE].

[55] ATLAS collaboration, Search for resonances decaying to photon pairs in $3.2 \mathrm{fb}^{-1}$ of $\mathrm{pp}$ collisions at $\sqrt{s}=13 \mathrm{TeV}$ with the ATLAS detector, ATLAS-CONF-2015-081.

[56] CMS collaboration, Search for new physics in high mass diphoton events in proton-proton collisions at $13 \mathrm{TeV}$, CMS-PAS-EXO-15-004.

[57] B. Grzadkowski, M. Iskrzynski, M. Misiak and J. Rosiek, Dimension-Six Terms in the Standard Model Lagrangian, JHEP 10 (2010) 085 [arXiv: 1008.4884] [INSPIRE].

[58] S.R. Coleman and E.J. Weinberg, Radiative Corrections as the Origin of Spontaneous Symmetry Breaking, Phys. Rev. D 7 (1973) 1888 [InSPIRE]. 